\title{
PERMITTED OXYGEN ABUNDANCES AND THE TEMPERATURE SCALE OF METAL-POOR TURNOFF STARS
}

\author{
JoRge MELÉndez ${ }^{1}$ \\ Department of Astronomy, California Institute of Technology, MC 105-24, Pasadena, CA 91125; and \\ Research School of Astronomy and Astrophysics, Mount Stromlo Observatory, \\ Cotter Road, Weston Creek, ACT 2611, Australia; jorge@mso.anu.edu.au \\ Nataliya G. Shchukina and Irina E. Vasiljeva \\ Main Astronomical Observatory, National Academy of Sciences, 27 Zabolotnogo Street, Kiev 03680, Ukraine; \\ shchukin@mao.kiev.ua, vasil@mao.kiev.ua \\ AND \\ IVÁN RAMíREZ ${ }^{1}$ \\ Department of Astronomy, University of Texas at Austin, RLM 15.306, TX 78712-1083; ivan@astro.as.utexas.edu \\ Received 2005 August 27; accepted 2006 January 11
}

\begin{abstract}
We use high-quality VLT/UVES published data of the permitted $\mathrm{O}_{\text {I }}$ triplet and Fe II lines to determine oxygen and iron abundances in unevolved (dwarfs, turnoff, subgiants) metal-poor halo stars. The calculations have been performed both in LTE and non-LTE (NLTE), employing effective temperatures obtained with the new infrared flux method (IRFM) temperature scale by Ramírez \& Meléndez, and surface gravities from Hipparcos parallaxes and theoretical isochrones. A new list of accurate transition probabilities for $\mathrm{Fe}$ II lines, tied to the absolute scale defined by laboratory measurements, has been used. Interstellar absorption has been carefully taken into account by employing reddening maps, stellar energy distributions and Strömgren photometry. We find a plateau in the oxygento-iron ratio over more than 2 orders of magnitude in iron abundance $(-3.2<[\mathrm{Fe} / \mathrm{H}]<-0.7)$, with a mean $[\mathrm{O} / \mathrm{Fe}]=0.5 \mathrm{dex}(\sigma=0.1 \mathrm{dex})$, independent of metallicity, temperature, and surface gravity. The flat $[\mathrm{O} / \mathrm{Fe}]$ ratio is mainly due to the use of adequate NLTE corrections and the new IRFM temperature scale, which, for metal-poor F/early G dwarfs is hotter than most $T_{\text {eff }}$ scales used in previous studies of the $\mathrm{O}$ I triplet. According to the new IRFM $T_{\text {eff }}$ scale, the temperatures of turnoff halo stars strongly depend on metallicity, a result that is in excellent qualitative and quantitative agreement with stellar evolution calculations, which predict that the $T_{\mathrm{eff}}$ of the turnoff at $[\mathrm{Fe} / \mathrm{H}]=-3$ is about $600-700 \mathrm{~K}$ higher than that at $[\mathrm{Fe} / \mathrm{H}]=-1$. Recent determinations of $\mathrm{H} \alpha$ temperatures in turnoff stars are in excellent relative agreement with the new IRFM $T_{\text {eff }}$ scale in the metallicity range $-2.7<$ $[\mathrm{Fe} / \mathrm{H}]<-1$, with a zero-point difference of only $61 \mathrm{~K}$.
\end{abstract}

Subject headings: atomic data — cosmic rays — Galaxy: halo — stars: abundances — stars: atmospheres stars: fundamental parameters - stars: Population II — Sun: abundances

Online material: color figures

\section{INTRODUCTION}

The importance of the oxygen abundance in metal-poor stars has been strongly emphasized in the literature, as well as the problems related to its determination. There is currently no consensus as to whether the $[\mathrm{O} / \mathrm{Fe}]$ ratio in halo stars is approximately constant $([\mathrm{O} / \mathrm{Fe}] \approx 0.4-0.5)$ or steeply increases with decreasing $[\mathrm{Fe} / \mathrm{H}]([\mathrm{O} / \mathrm{Fe}] \approx-0.35[\mathrm{Fe} / \mathrm{H}])$. The discrepancy is due to problems in the modeling of the different spectral features used to estimate the oxygen abundance, which are very hard to detect in the same star.

Due to its high excitation potential, the permitted $\mathrm{O}$ I triplet at $0.77 \mu \mathrm{m}$ is mainly observed in FG dwarfs and subgiants, being very faint (yet detectable) in metal-poor early $\mathrm{K}$ stars. The lowexcitation potential forbidden lines [O I] at $0.63 \mu \mathrm{m}$ are detected in giants and cool subgiants, and barely detectable in dwarfs. Molecular $\mathrm{OH}$ lines are observed in the ultraviolet (electronic transitions at $0.3 \mu \mathrm{m}$ ) and in the infrared (vibrational-rotational lines at 1.5 and $3 \mu \mathrm{m}$ ). Both observations are difficult: the UV

\footnotetext{
${ }^{1}$ Affiliated with the Seminario Permanente de Astronomía y Ciencias Espaciales of the Universidad Nacional Mayor de San Marcos, Peru.
}

$\mathrm{OH}$ lines are close to the atmospheric cutoff and most CCDs have low sensitivity in the UV, while the weak IR OH lines require extremely high $\mathrm{S} / \mathrm{N}$. The UV OH lines are relatively strong and observed in FGK metal-poor stars, while the very weak IR OH lines are only observed in cool stars with $T_{\text {eff }}<5000 \mathrm{~K}$.

The observed spectra by themselves do not allow a direct measurement of the oxygen abundance, so a careful modeling must be performed. Since the spectral features are sensitive to both effective temperature and surface gravity, reliable atmospheric parameters must be used. The analysis is complicated by the presence of both NLTE (mainly affecting the $\mathrm{O}_{\text {I }}$ triplet) and granulation (crucial for the molecular $\mathrm{OH}$ lines) effects.

Analyses of the forbidden [O I] (e.g., Barbuy 1988; Sneden et al. 1991) and infrared OH lines (Meléndez et al. 2001) seem to show a flat $[\mathrm{O} / \mathrm{Fe}]$, or a small increase of about $0.1-0.2$ dex per a factor of $10(1 \mathrm{dex})$ in metallicity, from $[\mathrm{O} / \mathrm{Fe}] \approx 0.3$ at $[\mathrm{Fe} / \mathrm{H}]=-1.5$ to $[\mathrm{O} / \mathrm{Fe}] \approx 0.4-0.5$ at $[\mathrm{Fe} / \mathrm{H}]=-2.5$ (Sneden \& Primas 2001; Meléndez \& Barbuy 2002). Yet, the onedimensional analysis of the forbidden line by Nissen et al. (2002) shows a continuous increase of $[\mathrm{O} / \mathrm{Fe}]$ for lower metallicities, reaching $[\mathrm{O} / \mathrm{Fe}] \approx+0.7$ at $[\mathrm{Fe} / \mathrm{H}]=-2.5$, but after correcting 
for three-dimensional effects they found a plateau at $[\mathrm{O} / \mathrm{Fe}] \approx$ +0.3 in the range $-2.0<[\mathrm{Fe} / \mathrm{H}]<-0.7$, and an increase in $[\mathrm{O} / \mathrm{Fe}]$ for lower $[\mathrm{Fe} / \mathrm{H}]$. The recent one-dimensional analysis of [O I] in subgiants by García Pérez et al. (2006) shows a mean $[\mathrm{O} / \mathrm{Fe}] \approx+0.5$, with a very small increase of $0.09 \mathrm{dex}$ in $[\mathrm{O} / \mathrm{Fe}]$ for a decrease of $1 \mathrm{dex}$ in $[\mathrm{Fe} / \mathrm{H}]$.

On the other hand, studies of the permitted O I triplet (e.g., Abia \& Rebolo 1989; Cavallo, Pilachowski \& Rebolo 1997; Israelian et al. 1998, 2001; Mishenina et al. 2000) and the ultraviolet OH lines (Israelian et al. 1998, 2001; Boesgaard et al. 1999) show a steep monotonic increase of $[\mathrm{O} / \mathrm{Fe}]$ for lower metallicities, reaching $[\mathrm{O} / \mathrm{Fe}]=+1.1$ at $[\mathrm{Fe} / \mathrm{H}]=-3$. However, other studies of the triplet show a lower (or zero) slope. For example, the analysis of the triplet in turnoff stars by Akerman et al. (2004, hereafter Ake04) shows only a mild increase of $[\mathrm{O} / \mathrm{Fe}]$ with metallicity, with $[\mathrm{O} / \mathrm{Fe}] \approx+0.4$ at $[\mathrm{Fe} / \mathrm{H}]=-1$ and $[\mathrm{O} / \mathrm{Fe}] \approx+0.7$ at $[\mathrm{Fe} / \mathrm{H}]=-3$.

Asplund \& García Pérez (2001) have shown that onedimensional model atmospheres overestimate the abundances obtained from UV OH lines by as much as $0.9 \mathrm{dex}$ at $[\mathrm{Fe} / \mathrm{H}]=$ -3 (see also $\S 8.2$ and Asplund 2006), and when the UV OH lines are analyzed with three-dimensional model atmospheres, the $[\mathrm{O} / \mathrm{Fe}]$ ratio in metal-poor dwarfs is in good agreement with the results obtained in giants from the forbidden lines. Note also that the one-dimensional analysis of the UV OH lines by Bessell et al. (1991) resulted in low $[\mathrm{O} / \mathrm{Fe}]$ ratios, as is also the case of the work on UV OH lines in subgiant stars by García Pérez et al. (2006), which shows one-dimensional oxygen-toiron ratios of $[\mathrm{O} / \mathrm{Fe}] \approx+0.5$ dex. Hence, analyses of three oxygen abundance features (UV OH lines, IR OH lines, [O I] lines) roughly agree in a somewhat flat (and low) $[\mathrm{O} / \mathrm{Fe}]$ ratio in the metallicity range $-3<[\mathrm{Fe} / \mathrm{H}]<-1$. However, the high oxygen abundances obtained from the $\mathrm{O}$ I triplet have been difficult to reconcile with the low oxygen abundance derived from the forbidden lines (Spite \& Spite 1991). Nissen et al. (2002) found that one-dimensional analysis results in agreement between the [O I] line and the triplet, but when the lines are analyzed employing three-dimensional model atmospheres the oxygen abundances obtained from the triplet are about 0.2 dex larger than those from the [O I] line (Nissen et al. 2002).

The disagreement between the $\mathrm{O}$ I triplet and the forbidden [O I] lines also occurs in metal-poor giants and subgiants (Cavallo et al. 1997; Fulbright \& Johnson 2003; García Pérez et al. 2006). Israelian et al. (2004) undertook the first NLTE analysis in very metal-poor CN-rich giants, showing that there are serious problems with standard one-dimensional model atmospheres of those stars, even when NLTE effects are taken into account, producing a serious conflict between the oxygen abundances obtained from the forbidden line and the triplet (Israelian et al. 2004). The use of $\mathrm{CN}$-enhanced model atmospheres have an important impact on the thermal structure of model atmospheres of metal-poor $\mathrm{CN}$-enhanced $([\mathrm{C} / \mathrm{Fe}]=+2,[\mathrm{~N} / \mathrm{Fe}]=+2)$ giants (Masseron et al. 2005), which affect the abundances from a few tenths of a dex up to 1.5 dex in extreme cases, with respect to standard (solar-scaled abundances) one-dimensional models (Masseron et al. 2005). Hence, for metal-poor cool giants with extreme compositions, model atmospheres computed with the corresponding CNO overabundances may partly relieve the problems found by Israelian et al. (2004).

The use of $3 \mathrm{D}+$ NLTE seems to improve the situation for the oxygen abundances derived from the $\mathrm{O}_{\mathrm{I}}$ triplet and the $\left[\mathrm{O}_{\mathrm{I}}\right]$ line. Employing 1.5 dimensional NLTE calculations in a threedimensional model atmosphere of the metal-poor star HD 140283, Shchukina et al. (2005) have found that the oxygen abundances obtained from the $\mathrm{O}_{\mathrm{I}}$ and $\left[\mathrm{O}_{\mathrm{I}}\right]$ lines agree within $\approx 0.1 \mathrm{dex}$, which is an important achievement considering the observational uncertainties for the [O I] line.

Previous studies of the $\mathrm{O}$ I triplet employing a hot temperature scale resulted in a flat and low [O/Fe] (King 1993; Carretta et al. 2000). Note that another reason why Carretta et al. (2000) obtained low $[\mathrm{O} / \mathrm{Fe}]$ ratios is the use of large NLTE corrections of the O I triplet by Gratton et al. (1999), as pointed out by Takeda (2003).

King (2000) studied the influence of stellar parameters and NLTE iron abundances (Thévenin \& Idiart 1999) on the [O/Fe] ratio obtained from $\mathrm{UV} \mathrm{OH},\left[\mathrm{O}_{\mathrm{I}}\right]$, and $\mathrm{O}_{\mathrm{I}}$ lines. The $[\mathrm{O} / \mathrm{Fe}]$ values obtained by King (2000) using the $\mathrm{O}_{\mathrm{I}}$ triplet and the UV OH lines are considerably reduced with respect to the high $[\mathrm{O} / \mathrm{Fe}]$ ratios of the original studies (Tomkin et al. 1992; Boesgaard et al. 1999), but they are still about $0.1-0.2$ dex larger than those obtained from the [O I] lines (King 2000). Partly the low [O/Fe] ratios obtained by King (2000) can be explained by the large NLTE Fe I corrections by Thévenin \& Idiart (1999), which increase the iron abundance by $+0.2-0.3$ dex, hence lowering $[\mathrm{O} / \mathrm{Fe}]$ by $0.2-0.3 \mathrm{dex}$.

The preliminary NLTE analysis of the $\mathrm{O}_{\mathrm{I}}$ triplet by Primas et al. (2001) also resulted in a flat $[\mathrm{O} / \mathrm{Fe}]$ ratio for halo dwarfs down to $[\mathrm{Fe} / \mathrm{H}]=-2.4$, independent of the $T_{\text {eff }}$ scale employed. Primas et al. found $[\mathrm{O} / \mathrm{Fe}] \approx+0.4$ using the $T_{\text {eff }}$ scale by Alonso et al. (1996), and $[\mathrm{O} / \mathrm{Fe}] \approx+0.5$ with both the $T_{\text {eff }}$ scale by Carney (1983) and temperatures from Thévenin \& Idiart (1999).

The observed $[\mathrm{O} / \mathrm{Fe}]$ ratio in halo stars provides tight constraints on models of Galaxy formation (e.g., Tinsley 1979; Wheeler et al. 1989; McWilliam 1997; Chiappini et al. 2003). If the formation of the halo was fast, then the $[\mathrm{O} / \mathrm{Fe}]$ ratio of halo stars should be roughly constant, because Type Ia supernovae, which originate from long-lived low-mass stars, would not have had enough time to lower the $[\mathrm{O} / \mathrm{Fe}]$ ratio of the interstellar medium (ISM) from which the halo stars we observe today were formed. Furthermore, the scatter of the $[\mathrm{O} / \mathrm{Fe}]$ ratio tells us the efficiency of the ISM in homogenizing (mixing) the ejecta of Type II supernovae (Scalo \& Elmegreen 2004).

The oxygen abundance is extremely important in studies of the production of LiBeB by Galactic cosmic-ray (GCR) spallation (e.g., Prantzos et al. 1993; Fields \& Olive 1999; Ramaty et al. 2000; Vangioni-Flam et al. 2000), especially for stars with $[\mathrm{Fe} / \mathrm{H}]<-2$, where current models struggle to explain the nonzero isotopic ${ }^{6} \mathrm{Li} /{ }^{7} \mathrm{Li}$ ratios recently found in metal-poor dwarfs down to $[\mathrm{Fe} / \mathrm{H}]=-2.7$ (Asplund et al. 2006). The GCR production of ${ }^{6} \mathrm{Li}$ depends on the adopted $[\mathrm{O} / \mathrm{Fe}]$ ratio, and only models with extremely high oxygen abundances can account for the ${ }^{6} \mathrm{Li}$ detection at $[\mathrm{Fe} / \mathrm{H}]=-2.7$, but introduce the problem of large overproduction of ${ }^{6} \mathrm{Li}$ at higher metallicities (Rollinde et al. 2005). Given the problems faced by GCR models to explain the ${ }^{6} \mathrm{Li}$ observed in halo stars, several authors have suggested that the origin of ${ }^{6} \mathrm{Li}$ may be pre-Galactic (Jedamzik 2000, 2004; Asplund et al. 2006; Rollinde et al. 2005). A low [O/Fe] ratio in metal-poor stars brings support for pre-Galactic ${ }^{6} \mathrm{Li}$, since in this case GCR cannot produce enough ${ }^{6} \mathrm{Li}$ (see also Prantzos 2006).

Here we analyze recent high-quality VLT/UVES data of the $\mathrm{O}$ I triplet and Fe II lines from Ake04 and Nissen et al. (2004, hereafter N04), respectively. We show that the use of NLTE corrections and the new infrared flux method (IRFM) temperature scale by Ramírez \& Meléndez (2005a, 2005b; hereafter RM05a and RM05b), which for metal-poor turnoff stars is hotter than previous temperature scales, results in a low and flat $[\mathrm{O} / \mathrm{Fe}]$ ratio in metal-poor stars. 
We also compare the IRFM temperature of turnoff stars (employing the new IRFM $T_{\text {eff }}$ scale) with those expected from stellar evolution, showing that the strong metallicity dependence of the turnoff temperatures are very well reproduced by stellar evolution models.

\section{SAMPLE}

Our sample stars consists of $31 \mathrm{~F}$ and $\mathrm{G}$ stars, which were selected by Ake04 and N04 to be close to the main-sequence turnoff and with halo kinematics. The equivalent widths of the $\mathrm{O}$ I triplet and $\mathrm{Fe}$ II lines were taken from Ake04 and N04, respectively. The superb VLT/UVES data were obtained at a resolving power $R=60,000$ with 4 pixels per spectral resolution element and a typical $\mathrm{S} / \mathrm{N}=200-300 \mathrm{pixel}^{-1}$ (Ake04; N04).

We have verified (within the uncertainties) that the sample stars have halo kinematics. We computed UVW velocities from their proper motions, radial velocities, and distances (mainly from Hipparcos parallaxes, but also from Strömgren photometry and isochrones), with data obtained from the SIMBAD database. The main uncertainty is due to the uncertainty in distance, which in some cases leads to an error of a few tens of $\mathrm{km} \mathrm{s}^{-1}$ in the UVW velocities.

\section{ATOMIC DATA AND SOLAR ABUNDANCES}

\subsection{The $\mathrm{O}$ I Triplet}

The oscillator strengths of the $\mathrm{O}_{\text {I }}$ triplet have been adopted from the NIST database (Wiese et al. 1996). We employed an interaction broadening constant $C_{6}\left[\mathrm{O}_{\mathrm{I}}\right]=0.84 \times 10^{-31}$, obtained from the collision broadening cross sections given by Barklem et al. (2000). Our O I atomic model for the NLTE calculations is based on data by Carlsson \& Judge (1993). The NLTE spectrum synthesis code NATAJA was employed in the present study. For details see Shchukina et al. $(2003,2005)$ and Shchukina \& Trujillo Bueno (2001).

Equivalent widths $\left(W_{\lambda}\right)$ of the $\mathrm{O}_{\mathrm{I}}$ triplet have been measured employing the National Solar Observatory (NSO) FTS solar flux spectrum by Hinkle et al. (2000), which is essentially the same spectrum previously published by Kurucz et al. (1984, hereafter K84), but corrected for telluric absorption at $\lambda>5000 \AA$. We have checked that the NSO FTS data are in the same scale as those of the VLT/UVES data, using a high-resolution $\left(R \approx 10^{5}\right)$ UVES reflected solar spectrum. ${ }^{2}$ As shown in Table 1 , both measurements are in excellent agreement (see $\S 3.2$ for a comparison between $\mathrm{Fe}$ II lines), and they also agree very well with the predicted $W_{\lambda}$ of the $\mathrm{O}_{\mathrm{I}}$ triplet given by Asplund et al. (2004, hereafter Asp04).

Employing a Kurucz overshooting model atmosphere (Castelli et al. 1997) and $W_{\lambda}$ from both the K84 (Hinkle et al. 2000) and VLT/UVES solar spectrum we found $A(\mathrm{O})=8.65(\sigma=0.03)$. This is in very good agreement to other recent NLTE determinations of the oxygen abundance using the $\mathrm{O}$ I triplet. For example, Meléndez (2004) found $A(\mathrm{O})=8.68$ employing a spatially and temporally averaged three-dimensional solar model, and $A(\mathrm{O})=$ 8.67 with a Kurucz solar model. Full 3D+NLTE calculations by Asp04 and Allende Prieto et al. (2004) resulted in $A(\mathrm{O})=8.64$ and 8.70 , respectively, while $A(\mathrm{O})=8.70$ was obtained by Shchukina et al. (2005) with 1.5 dimensional NLTE calculations in a three-dimensional solar model (Asplund et al. 2000). The recommended solar oxygen abundance by Asp04 is $8.66 \pm 0.05$, which is based on three-dimensional analyses of the forbidden, permitted and infrared $\mathrm{OH}$ lines of the $\Delta v=0,1$ sequences.
TABLE 1

O I TRIPLET

\begin{tabular}{ccccccc}
\hline \hline & & & & \multicolumn{3}{c}{$W_{\lambda \odot}$} \\
\cline { 4 - 6 }$\lambda$ & & & & & \\
\cline { 5 - 7 }$(\AA)$ & & $(\mathrm{eV})$ & $\begin{array}{c}\log g f \\
(\mathrm{dex})\end{array}$ & $\begin{array}{c}\mathrm{K} 84 \\
(\mathrm{~m} \AA)\end{array}$ & $\begin{array}{c}\text { UVES } \\
(\mathrm{m} \AA)\end{array}$ & $\begin{array}{c}\text { Asp04 } \\
(\mathrm{m} \AA)\end{array}$ \\
\hline $7771.944 \ldots \ldots \ldots$. & 9.1461 & 0.37 & 71.5 & 72.0 & 71.2 \\
$7774.166 \ldots \ldots \ldots .$. & 9.1461 & 0.22 & 61.9 & 61.8 & 61.8 \\
$7775.388 \ldots \ldots \ldots .$. & 9.1461 & 0.00 & 48.6 & 47.4 & 48.8 \\
\hline
\end{tabular}

If we also consider the solar oxygen abundance obtained from the first-overtone $\mathrm{OH}$ lines, then the weighted mean solar $\mathrm{O}$ abundance is 8.64 (Meléndez 2004). The value adopted in this work is $A(\mathrm{O})_{\odot}=8.65$.

\subsection{Fe II Lines}

As previously discussed in the literature (e.g., Lambert et al. 1996; Asplund et al. 2000; Gehren et al. 2001), there is a lack of accurate experimental $g f$-values for Fe II lines. With the advent of 8-10 m telescopes and very efficient spectrographs, high$\mathrm{S} / \mathrm{N}$ high spectral resolution data can be readily obtained, and therefore one of the main limitations for accurate stellar abundance work is the uncertainty in the transition probabilities of Fe II lines.

In the present work the $g f$-values of $\mathrm{Fe}$ II lines are from a revised version of the list of Meléndez \& Barbuy (2002), where relative $g f$-values within a given multiplet were taken from theoretical determinations and the absolute scale of the transition probabilities of each multiplet was determined from laboratory lifetimes and branching ratios. The approach of Meléndez \& Barbuy (2002) has the advantage of improving the accuracy of the $g f$-values while preserving the laboratory scale of the oscillator strengths. We stress here that a single correction factor cannot be applied to the whole set of theoretical $g f$-values, since the theoretical calculations should only be reliable within a single multiplet. This is why each multiplet needs to be corrected individually, in order to put the whole set of multiplets into the absolute laboratory scale.

The revised Fe II line list takes into account new laboratory measurements by Schnabel et al. (2004) and new theoretical calculations by R. L. Kurucz. ${ }^{3}$ Further details and the complete list of Fe II lines for the optical region will be presented in J. Meléndez $\&$ B. Barbuy (2006, in preparation). The subset of $g f$-values used for the solar and stellar analysis are given in Table 2 . The interaction broadening constants $\left(C_{6}\right)$ of the Fe II lines were obtained from the cross sections recently computed by Barklem \& AspelundJohansson (2005).

As an example of the quality of the new $g f$-values, in Figure 1 are shown the solar iron abundances obtained from Fe II lines by Hannaford et al. (1992, hereafter H92), and the rescaled abundances $\left[A(\mathrm{Fe})^{\text {new }}=A(\mathrm{Fe})^{\mathrm{H} 92}+\log g f^{\mathrm{H} 92}-\log g f^{\text {new }}\right]$ due to the new $g f$-values given in the present work. As can be seen, significant progress has been achieved, reducing the scatter from $\sigma=0.07 \mathrm{dex}(g f$-values from H92) to $\sigma=0.03 \mathrm{dex}$ (new $g f$-values). The mean solar iron abundance from H92 is 7.47 , and with the improved $g f$-values is 7.46. Similar comparisons with other works in the literature also show that our new $g f$-values lead to a decrease in the line-to-line scatter of the solar iron abundance (J. Meléndez \& B. Barbuy 2006, in preparation).

2 See http://www.eso.org/observing/dfo/quality/UVES.

3 See http://kurucz.harvard.edu. 


\begin{tabular}{|c|c|c|c|c|}
\hline \multirow[b]{2}{*}{$\begin{array}{c}\lambda \\
(\AA)\end{array}$} & \multirow[b]{2}{*}{$\begin{array}{l}\chi_{\mathrm{exc}} \\
(\mathrm{eV})\end{array}$} & \multirow[b]{2}{*}{$\begin{array}{r}\log g f \\
(\mathrm{dex})\end{array}$} & \multicolumn{2}{|c|}{$W_{\lambda \odot}$} \\
\hline & & & K84 & $\begin{array}{l}\text { UVES } \\
(\mathrm{m} \AA)\end{array}$ \\
\hline $4128.748^{\mathrm{a}}$. & 2.5828 & -3.63 & & $\ldots$ \\
\hline $4178.862^{\mathrm{a}} .$. & 2.5828 & -2.51 & $\ldots$ & $\ldots$ \\
\hline $4233.172^{\mathrm{a}} \ldots \ldots \ldots \ldots$ & 2.5828 & -1.97 & $\ldots$ & $\ldots$ \\
\hline 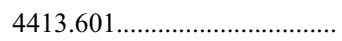 & 2.6759 & -3.79 & 38.3 & 39.5 \\
\hline $4416.830^{\mathrm{a}}$ & 2.7786 & -2.65 & $\ldots$ & $\ldots$ \\
\hline $4489.183^{\mathrm{a}} \ldots \ldots \ldots$ & 2.8283 & -2.96 & $\ldots$ & $\ldots$ \\
\hline $4491.405^{\mathrm{a}}$. & 2.8557 & -2.71 & 69.0 & 70.6 \\
\hline $4508.288^{\mathrm{a}}$. & 2.8557 & -2.44 & 83.5 & 82.9 \\
\hline $4515.339^{\mathrm{a}}$. & 2.8443 & -2.60 & $\ldots$ & $\ldots$ \\
\hline $4520.224^{\mathrm{a}}$. & 2.8068 & -2.65 & 75.0 & 76.1 \\
\hline $4522.634^{\mathrm{a}}$. & 2.8443 & -2.25 & $\ldots$ & $\ldots$ \\
\hline $4534.168 \ldots$ & 2.8557 & -3.28 & 51.3 & 51.9 \\
\hline $4541.524^{\mathrm{a}}$. & 2.8557 & -2.98 & 61.0 & 60.6 \\
\hline $4555.893^{\mathrm{a}}$ & 2.8283 & -2.40 & 86.5 & 84.3 \\
\hline $4576.340^{\mathrm{a}, \mathrm{b}} \ldots \ldots \ldots$ & 2.8443 & -2.95 & 63.5 & 62.5 \\
\hline $4582.835^{\mathrm{a}} \ldots \ldots \ldots \ldots$ & 2.8443 & -3.18 & 52.9 & 52.9 \\
\hline $4583.837^{\mathrm{a}} \ldots$ & 2.8068 & -1.93 & $\ldots$ & $\ldots$ \\
\hline $4620.521^{\mathrm{a}, \mathrm{b}}$ & 2.8283 & -3.21 & 49.5 & 51.2 \\
\hline $4656.981^{\mathrm{a}, \mathrm{b}}$ & 2.8912 & -3.60 & 33.1 & 31.1 \\
\hline $4666.758^{\mathrm{a}} \ldots$ & 2.8283 & -3.28 & 47.0 & 48.6 \\
\hline $4923.927^{\mathrm{a}} \ldots$ & 2.8912 & -1.26 & $\ldots$ & $\ldots$ \\
\hline $5197.577 \ldots \ldots$ & 3.2306 & -2.22 & 79.9 & 81.8 \\
\hline $5234.625^{\mathrm{b}}$. & 3.2215 & -2.18 & 81.0 & 83.2 \\
\hline $5264.812^{\mathrm{b}} .$. & 3.2304 & -3.13 & 46.1 & 45.4 \\
\hline $5325.553 \ldots \ldots \ldots \ldots \ldots \ldots \ldots \ldots$ & 3.2215 & -3.16 & 42.1 & 41.2 \\
\hline $5414.073^{\mathrm{b}} \ldots \ldots \ldots \ldots \ldots \ldots$ & 3.2215 & -3.58 & 26.5 & 25.6 \\
\hline $5425.257 \ldots \ldots \ldots \ldots$ & 3.1996 & -3.22 & 40.7 & 41.6 \\
\hline $6369.462 \ldots \ldots \ldots$ & 2.8912 & -4.11 & 18.7 & 18.3 \\
\hline $6432.680^{\mathrm{b}}$. & 2.8912 & -3.57 & 41.3 & 40.7 \\
\hline $6516.080^{\mathrm{b}}$. & 2.8912 & -3.31 & 53.3 & 52.5 \\
\hline $7222.394^{\mathrm{b}}$. & 3.8889 & -3.26 & 19.4 & 18.5 \\
\hline $7224.487^{\mathrm{b}}$. & 3.8891 & -3.20 & 18.9 & 18.9 \\
\hline $7449.335^{\mathrm{b}}$. & 3.8889 & -3.27 & 17.1 & 18.2 \\
\hline $7515.832^{\mathrm{b}}$. & 3.9036 & -3.39 & 13.3 & 13.5 \\
\hline $7711.724^{\mathrm{b}}$ & 3.9034 & -2.50 & 45.9 & 46.7 \\
\hline
\end{tabular}

${ }^{\mathrm{a}}$ Line used for halo star.

${ }^{\mathrm{b}}$ Line in common with $\mathrm{H} 92$.

It is also interesting to compare our new $g f$-values of Fe II lines with the solar $g$-values determined by Gurtovenko \& Kostik (1989, hereafter GK89), which should be reliable in a relative scale, hence allowing us to check our relative (theoretical) oscillator strengths. A comparison between 20 lines in common shows an excellent relative agreement, with a $\sigma=0.038 \mathrm{dex}$, but with a systematic difference of 0.18 dex between both sets, that is mainly due to the high solar Fe abundance adopted by GK89.

In order to determine the solar iron abundance using our new $g f$-values of $\mathrm{Fe}$ II lines, we selected $\mathrm{Fe}$ II lines with the cleanest profiles in the NSO FTS solar flux spectrum (Hinkle et al. 2000; K84). As previously noted by H92, the lines at 5525.1 and $5627.5 \AA$ are significantly perturbed at the wings. H92 included these lines by fitting Gaussian line shapes; however, we discarded them because the deblended profiles were significantly asymmetric. Our measured $W_{\lambda}$ from the NSO solar flux spectrum, which are reported in Table 2, are in excellent agreement with our measurements from the VLT/UVES reflected solar spectrum (§3.1), with a mean difference (K84-UVES) of only $-0.1 \pm 1.2 \mathrm{~m} \AA(=-0.1 \% \pm 2.8 \%)$.

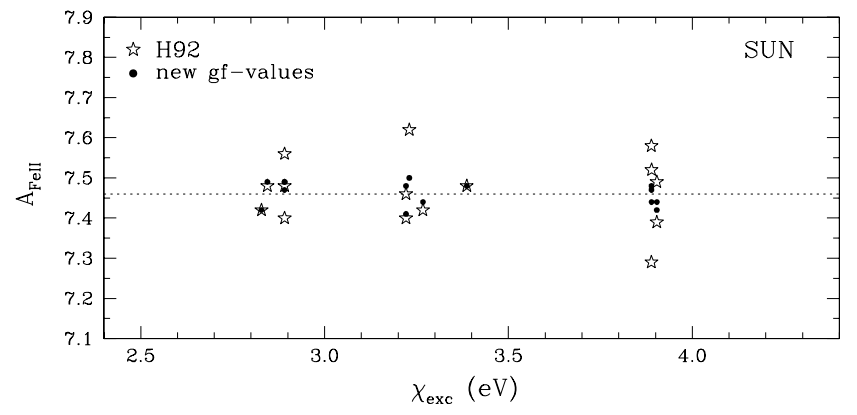

FIG. 1.-Solar iron abundances from Fe II lines vs. excitation potential (H92, stars), and rescaled abundances employing our new $g$-values ( filled circles). The scatter has been significantly reduced due to our improved oscillator strengths.

Our Fe $\mathrm{I}+\mathrm{Fe}_{\text {II }}$ atomic model for the NLTE calculations includes over 250 levels and nearly 500 radiative transitions. The model is similar to that used by Shchukina \& Trujillo Bueno (2001) and Shchukina et al. (2003, 2005).

Employing the Kurucz model atmosphere we obtained a solar iron abundance $A(\mathrm{Fe} \text { II })_{\mathrm{NLTE}}=A(\mathrm{Fe} \text { II })_{\mathrm{LTE}}=7.45(\sigma=0.04)$. This value is in good agreement with $1.5 \mathrm{D}+\mathrm{NLTE}$ calculations in a three-dimensional solar model (Asplund et al. 2000) by Shchukina \& Trujillo Bueno (2001), who found $A\left(\mathrm{Fe}_{\mathrm{I}}\right)=7.50$, and also in good agreement with previous one- and threedimensional results from $\mathrm{Fe}$ II lines by $\mathrm{H} 92$, Schnabel et al. (1999), and Asplund et al. (2000), who obtained $A(\mathrm{Fe}$ II) $=7.47$ (one-dimensional), 7.42 (one-dimensional) and 7.45 (threedimensional), respectively. We adopted $A(\mathrm{Fe} \text { II })_{\odot}=7.45$.

We end this section by showing the effect of different model atmospheres on the solar iron abundance and its $\sigma$, employing 3 different sets of $g f$-values for $13 \mathrm{Fe}$ II lines in common between the present work, H92 and GK89. The calculations were performed using the same set of input equivalent widths (Table 2) and $v_{t}=0.9 \mathrm{~km} \mathrm{~s}^{-1}$. We employed the Kurucz overshooting model, a MARCS model (Asplund et al. 1997), a spatially and temporally averaged three-dimensional solar model atmosphere ( $\langle 3 \mathrm{D}\rangle$; Asp04), and the Holweger \& Müller (1974, hereafter HM74) solar model. The results are shown in Table 3. As can be seen, the $\mathrm{Fe}$ abundance obtained from Fe II lines has only a small dependence on the adopted model atmosphere. A line-toline abundance scatter of $\sigma \approx 0.05 \mathrm{dex}$ is obtained for our $g f$-scale and the solar $g f$-scale of GK89, while the worst scatter ( $0.11 \mathrm{dex})$ is obtained with the $\mathrm{H} 92 \mathrm{gf}$-values. Certainly, $\sigma$ cannot be used as the only criterion for the quality of a $g f$-scale, since the scatter depends on both the adopted model atmosphere (see Table 3) and $v_{t}$ (see Gehren et al. (2001) and Kostik et al. (1996) for

TABLE 3

Solar Fe Abundance and $\sigma^{\text {a }}$ from Different Sets of Fe il Lines ${ }^{\text {b }}$

\begin{tabular}{|c|c|c|c|}
\hline \multirow[b]{2}{*}{ Model AtMosphere } & \multicolumn{3}{|c|}{$g f$-VALUES } \\
\hline & H92 & This Work & $\mathrm{GK} 89^{\mathrm{c}}$ \\
\hline Kurucz.... & $7.46(0.11)$ & $7.45(0.05)$ & $7.63(0.05)$ \\
\hline MARCS & $7.43(0.11)$ & $7.42(0.06)$ & $7.60(0.06)$ \\
\hline$\langle 3 \mathrm{D}\rangle$ & $7.44(0.11)$ & $7.43(0.05)$ & $7.61(0.06)$ \\
\hline 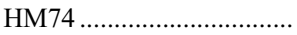 & $7.46(0.11)$ & $7.45(0.05)$ & $7.63(0.04)$ \\
\hline
\end{tabular}

${ }^{\text {a }}$ Value of $\sigma$ is given in parenthesis.

b We used $13 \mathrm{Fe}$ II lines in common between this work (Table 2), H92, and GK89

${ }^{\mathrm{c}}$ Solar oscillator strengths. 
details on the effects of $v_{t}$ ), but a good $g f$-scale should give a small $\sigma$ in order to give consistent results when only few lines are available for analysis.

\section{REDDENING}

Before colors are used to determine $T_{\text {eff }}$, they have to be corrected for interstellar absorption. A good determination of the reddening is specially important for metal-poor turnoff stars due to the steep slopes of their temperature versus color relations. $E_{B-V}$ values were estimated employing (1) several reddening maps $\left(E_{B-V}^{\text {maps }}\right),(2)$ relative stellar flux distributions $\left(E_{B-V}^{\mathrm{SED}}\right)$, and (3) Strömgren photometry $\left(E_{B-V}^{\text {uvby }-\beta}\right)$, as given by N04. Each method is described below.

\subsection{Reddening Maps}

Several studies show that nearby stars closer than $75 \mathrm{pc}$ have negligible reddening, since most stars within this distance are located inside the "Local Bubble" of radius $\approx 70-75 \mathrm{pc}$ (e.g., Lallement et al. 2003; Breitschwerdt et al. 2000; Sfeir et al. 1999; Leroy 1999; Vergely et al. 1998). ${ }^{4}$

We consider several extinction surveys (e.g., Fitzgerald 1968; Neckel \& Klare 1980; Arenou et al. 1992, hereafter A92) included in a Fortran code by Hakkila et al. (1997, hereafter H97), adopting the weighted-average with the inverse square of the errors as weights. The A92 extinction model included in the H97 code seems to systematically overestimate the reddening for stars with a distance $d<0.5 \mathrm{kpc}$, as shown by Chen et al. (1998, hereafter $\mathrm{C} 98$ ), who found that the average extinction derived from Strömgren photometry is about $40 \%$ lower than the average extinction derived from the A92 maps. Instead of discarding $E_{B-V}^{A 92}$ values for stars closer than $500 \mathrm{pc}$, we doubled its error (equivalent to lower its weight by a factor of 4) and corrected $E_{B-V}^{A 92}$ by a factor of 0.6 for stars in this distance range, as suggested by the results of $\mathrm{C} 98$.

We also employ the empirical reddening laws by Bond (1980) and C98, which are both cosecant laws dependent on distance and Galactic latitude, although for low-latitude $\left(|b| \leq 10^{\circ}\right)$ objects closer than $1 \mathrm{kpc}$ C98 law also includes a dependence with Galactic longitude.

Reddening corrections were also obtained from the $E_{B-V}$ map by Schlegel et al. (1998, hereafter S98), which is based on Cosmic Background Explorer Diffuse Infrared Background Experiment (COBE DIRBE) and Infrared Astronomical Satellite (IRAS) observations. Note that this map seems to systematically overestimate the reddening by about $20 \%$ (e.g., Arce \& Goodman 1999; Chen et al. 1999; Dutra et al. 2003a, 2003b), and some studies argue for a systematic zero-point error in S98 map. For example, Hudson (1999) found that the $E_{B-V}$ obtained from S98 is about 0.016 mag higher than the reddening of $86 \mathrm{RR}$ Lyrae given by Burstein \& Heiles (1978). We have made the same comparison for the sample of RR Lyrae given in Hudson (1999), and have found that indeed for $E_{B-V}<0.2, E_{B-V}^{S 98}$ should be corrected by $-0.02 \mathrm{mag}$. Burstein (2003) confirmed the existence of a zero-point difference of -0.02 mag between the maps of S98 and those from Burstein \& Heiles (1978).

We adopted the following correction for the S98 maps:

$$
E_{B-V}^{S 98 c}=0.9 E_{B-V}^{S 98}-0.01 .
$$

\footnotetext{
${ }^{4}$ Note, however, that the Local Bubble is by no means spherical, but roughly within $75 \mathrm{pc}$ of the Sun the extinction is negligible, although in some cases even stars as far as $100 \mathrm{pc}$ can be almost unreddened.
}

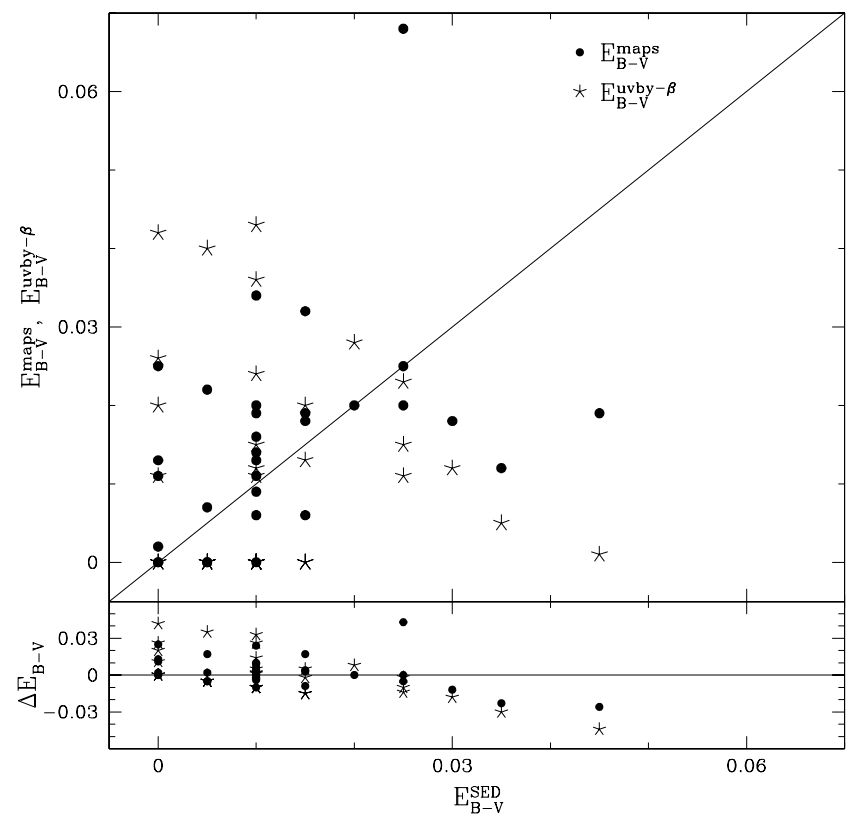

FIG. 2.-Comparison between extinction obtained from maps $\left(E_{B-V}^{\text {maps }}\right)$, Strömgren photometry $\left(E_{B-V}^{\mathrm{uvby}-\beta}\right)$, and stellar energy distributions $\left(E_{B-V}^{\mathrm{SED}}\right)$.

The different prescriptions for the determination of $E_{B-V}^{\text {maps }}$ were combined as follows:

1. $E_{B-V}^{\text {maps }}=0.0$ for stars within $75 \mathrm{pc}$.

2. For stars with $75 \mathrm{pc}<d \leq 100 \mathrm{pc}, E_{B-V}^{\text {maps }}=\left(E_{B-V}^{H 97}+\right.$ $\left.\left(E_{B-V}^{B 80}+E_{B-V}^{C 98}\right) / 2+2 \times 0.0\right) / 4$. The factor $2 \times 0.0$ considers a $50 \%$ chance of zero reddening.

3. If a star has $d>100 \mathrm{pc}$ and $|b|>45^{\circ}, E_{B-V}^{\text {maps }}=\left(n E_{B-V}^{H 97}+\right.$ $\left.m E_{B-V}^{S 98 c}\right) /(n+m)$, where $n$ is the square root of the number of maps used in $\mathrm{H} 97$ and $m=(d+1.5)(\sin |b|)^{1.5}$, with $d$ in kpc. This empirical parameterization gives a high weight to the S98 map for high-latitude and/or distant objects, and a weight essentially zero for low-latitude stars.

4. Stars with $d>100 \mathrm{pc}$ and $|b| \leq 45^{\circ}, E_{B-V}^{\text {maps }}=\left(n E_{B-V}^{H 97}+\right.$ $\left.m E_{B-V}^{S 98 c}+\left(E_{B-V}^{B 80}+E_{B-V}^{C 98}\right) / 2\right) /(n+m+1)$. The weights $n$ and $m$ are equal to the previous case, except that $m=0$ if $E_{B-V}^{S 98 c}>$ $2 E_{B-V}^{H 97}$ (in some cases this restriction was relaxed, especially for very distant objects with $|b|>30^{\circ}$ ).

5. If after applying the above criteria $E_{B-V}^{\text {maps }}>E_{B-V}^{S 98 c}$, then we adopted $E_{B-V}^{\text {maps }}=E_{B-V}^{S 98 c}$.

The distances employed here were estimated from Hipparcos parallaxes, Strömgren photometry (N04), and isochrones (see $\S 6)$.

\subsection{Extinction from Stellar Energy Distributions}

The observed relative spectral energy distribution (SED) of a star is defined primarily by its $T_{\text {eff }}$ and by interstellar extinction. The right $E_{B-V}$ value can be used to correct the observed SED, recovering thus the unredenned SED. Hence, given several observed colors $(X-Y)_{i}$ and highly accurate color versus $T_{\text {eff }}$ relations, the minimum scatter between different color temperatures $\left[T_{\text {eff }}(X-Y)_{i}\right]$ should be obtained for the right choice of $E_{B-V}$, denoted by $E_{B-V}^{\mathrm{SED}}$.

We employed our IRFM $T_{\text {eff }}$ scale (RM05b), which includes 17 colors from the blue to the near infrared, allowing for a good estimate of $E_{B-V}^{\mathrm{SED}}$. Considering that our $T_{\text {eff }}$ calibrations have high internal accuracy, the main uncertainty in the determination of $E_{B-V}^{\mathrm{SED}}$ arises from photometric errors. We typically employed 
TABLE 4

Reddening, Atmospheric Parameters and Chemical Abundances

\begin{tabular}{|c|c|c|c|c|c|c|c|c|c|c|c|c|}
\hline \multirow[b]{2}{*}{$\begin{array}{l}\text { ID } \\
\text { (1) }\end{array}$} & \multirow[b]{2}{*}{$\begin{array}{c}E_{B-V} \\
\text { (mag) } \\
\text { (2) }\end{array}$} & \multirow[b]{2}{*}{$\begin{array}{l}T_{\text {eff }} \\
(\mathrm{K}) \\
(3)\end{array}$} & \multirow[b]{2}{*}{$\begin{array}{c}\log g \\
\text { (dex) } \\
\text { (4) }\end{array}$} & \multirow[b]{2}{*}{$\begin{array}{c}{[\mathrm{M} / \mathrm{H}]} \\
(\mathrm{dex}) \\
(5)\end{array}$} & \multirow[b]{2}{*}{$\begin{array}{c}v_{t} \\
\left(\mathrm{~km} \mathrm{~s}^{-1}\right) \\
(6)\end{array}$} & \multicolumn{3}{|c|}{$\mathrm{Fe}$ II } & \multicolumn{3}{|c|}{$\mathrm{O}_{\mathrm{I}}$} & \multirow[b]{2}{*}{$\begin{array}{c}{[\mathrm{O} / \mathrm{Fe}]} \\
(\mathrm{dex}) \\
(13)\end{array}$} \\
\hline & & & & & & $\begin{array}{l}A_{\mathrm{LTE}} \\
(\mathrm{dex}) \\
(7)\end{array}$ & $\begin{array}{c}A_{\mathrm{NLTE}} \\
(\mathrm{dex}) \\
(8)\end{array}$ & $\begin{array}{c}{[\mathrm{Fe} / \mathrm{H}]_{\mathrm{NLTE}}{ }^{\mathrm{a}}} \\
(\mathrm{dex}) \\
(9)\end{array}$ & $\begin{array}{l}A_{\mathrm{LTE}} \\
(\mathrm{dex}) \\
(10)\end{array}$ & $\begin{array}{c}A_{\mathrm{NLTE}} \\
\text { (dex) } \\
(11)\end{array}$ & $\begin{array}{c}{[\mathrm{O} / \mathrm{H}]_{\mathrm{NLTE}}{ }^{\mathrm{a}}} \\
\quad(\mathrm{dex}) \\
\quad(12)\end{array}$ & \\
\hline $\mathrm{BD}+023375 \ldots \ldots \ldots \ldots$ & $0.022 \pm 0.022$ & $6045 \pm 69$ & $4.18 \pm 0.12$ & -2.0 & 1.5 & 5.26 & 5.32 & $-2.13 \pm 0.08$ & 7.43 & 7.25 & $-1.40 \pm 0.12$ & $0.73 \pm 0.18$ \\
\hline $\mathrm{BD}+024651 \ldots \ldots \ldots$ & $0.019 \pm 0.007$ & $6132 \pm 58$ & $3.85 \pm 0.15$ & -1.6 & 1.8 & 5.71 & 5.76 & $-1.69 \pm 0.07$ & 7.74 & 7.53 & $-1.12 \pm 0.02$ & $0.57 \pm 0.09$ \\
\hline BD +08 $3095 \ldots \ldots \ldots \ldots$ & $0.012 \pm 0.012$ & $5657 \pm 41$ & $4.21 \pm 0.30$ & -0.6 & 1.3 & 6.72 & 6.75 & $-0.70 \pm 0.10$ & 8.75 & 8.48 & $-0.17 \pm 0.02$ & $0.53 \pm 0.13$ \\
\hline $\mathrm{BD}+174708 \ldots \ldots \ldots$ & $0.009 \pm 0.008$ & $6091 \pm 92$ & $4.01 \pm 0.16$ & -1.4 & 1.8 & 5.86 & 5.92 & $-1.53 \pm 0.08$ & 7.95 & 7.72 & $-0.93 \pm 0.05$ & $0.60 \pm 0.13$ \\
\hline BD $-043208 \ldots \ldots \ldots \ldots$ & $0.013 \pm 0.013$ & $6376 \pm 59$ & $3.84 \pm 0.15$ & -2.1 & 1.5 & 5.17 & 5.20 & $-2.25 \pm 0.07$ & 7.17 & 6.98 & $-1.67 \pm 0.03$ & $0.58 \pm 0.11$ \\
\hline BD -13 $3442 \ldots \ldots \ldots \ldots$ & $0.018 \pm 0.021$ & $6510 \pm 40$ & $4.10 \pm 0.30$ & -2.5 & 1.5 & 4.89 & 4.92 & $-2.53 \pm 0.06$ & 6.89 & 6.67 & $-1.98 \pm 0.07$ & $0.55 \pm 0.13$ \\
\hline $\mathrm{CD}-3018140 \ldots \ldots \ldots \ldots$ & $0.023 \pm 0.004$ & $6305 \pm 44$ & $4.28 \pm 0.12$ & -1.7 & 1.9 & 5.63 & 5.68 & $-1.77 \pm 0.08$ & 7.66 & 7.46 & $-1.19 \pm 0.04$ & $0.58 \pm 0.10$ \\
\hline $\mathrm{CD}-3514849 \ldots \ldots \ldots \ldots$ & $0.016 \pm 0.003$ & $6313 \pm 43$ & $4.33 \pm 0.15$ & -2.0 & 1.5 & 5.17 & 5.21 & $-2.24 \pm 0.07$ & 7.11 & 6.95 & $-1.70 \pm 0.06$ & $0.54 \pm 0.10$ \\
\hline CD $-4214278 \ldots \ldots \ldots \ldots$ & $0.020 \pm 0.009$ & $6006 \pm 42$ & $4.52 \pm 0.15$ & -1.8 & 1.4 & 5.51 & 5.59 & $-1.86 \pm 0.07$ & 7.41 & 7.25 & $-1.40 \pm 0.02$ & $0.46 \pm 0.09$ \\
\hline 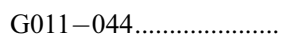 & $0.005 \pm 0.005$ & $6092 \pm 47$ & $4.49 \pm 0.15$ & -1.9 & 1.5 & 5.49 & 5.56 & $-1.89 \pm 0.07$ & 7.52 & 7.35 & $-1.30 \pm 0.04$ & $0.59 \pm 0.09$ \\
\hline G024-003 ................. & $0.039 \pm 0.025$ & $6077 \pm 35$ & $4.41 \pm 0.15$ & -1.4 & 1.6 & 5.92 & 5.98 & $-1.47 \pm 0.08$ & 7.67 & 7.49 & $-1.16 \pm 0.06$ & $0.31 \pm 0.15$ \\
\hline G053-041_......................... & $0.017 \pm 0.015$ & $5970 \pm 27$ & $4.38 \pm 0.15$ & -1.1 & 1.4 & 6.24 & 6.29 & $-1.16 \pm 0.08$ & 7.89 & 7.71 & $-0.94 \pm 0.02$ & $0.22 \pm 0.11$ \\
\hline G064-012 $\ldots \ldots \ldots \ldots \ldots$ & $0.017 \pm 0.019$ & $6660 \pm 77$ & $4.24 \pm 0.15$ & -3.0 & 1.5 & 4.26 & 4.28 & $-3.17 \pm 0.10$ & 6.36 & 6.01 & $-2.64 \pm 0.11$ & $0.53 \pm 0.18$ \\
\hline 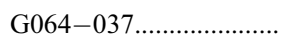 & $0.010 \pm 0.001$ & $6658 \pm 61$ & $4.27 \pm 0.15$ & -2.8 & 1.5 & 4.44 & 4.46 & $-2.99 \pm 0.06$ & 6.35 & 6.02 & $-2.63 \pm 0.04$ & $0.36 \pm 0.09$ \\
\hline 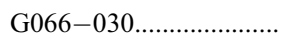 & $0.016 \pm 0.007$ & $6316 \pm 38$ & $4.18 \pm 0.15$ & -1.3 & 1.8 & 5.91 & 5.95 & $-1.50 \pm 0.08$ & 7.99 & 7.74 & $-0.91 \pm 0.02$ & $0.59 \pm 0.10$ \\
\hline HD 103723 & $0.020 \pm 0.013$ & $6013 \pm 21$ & $4.26 \pm 0.12$ & -0.6 & 1.4 & 6.66 & 6.69 & $-0.76 \pm 0.10$ & 8.41 & 8.14 & $-0.51 \pm 0.02$ & $0.25 \pm 0.12$ \\
\hline HD $106038 \ldots \ldots \ldots \ldots \ldots \ldots . . .$. & $0.007 \pm 0.006$ & $6012 \pm 26$ & $4.45 \pm 0.11$ & -1.2 & 1.7 & 6.08 & 6.14 & $-1.31 \pm 0.08$ & 8.11 & 7.90 & $-0.75 \pm 0.03$ & $0.56 \pm 0.10$ \\
\hline HD $108177 \ldots \ldots \ldots \ldots$ & $0.003 \pm 0.005$ & $6133 \pm 49$ & $4.42 \pm 0.1^{\mathrm{b}}$ & -1.5 & 1.8 & 5.81 & 5.86 & $-1.59 \pm 0.08$ & 7.85 & 7.65 & $-1.00 \pm 0.04$ & $0.59 \pm 0.10$ \\
\hline HD 110621.......................... & $0.013 \pm 0.004$ & $6083 \pm 51$ & $4.01 \pm 0.12$ & -1.4 & 1.7 & 5.84 & 5.88 & $-1.57 \pm 0.08$ & 7.95 & 7.73 & $-0.92 \pm 0.08$ & $0.65 \pm 0.12$ \\
\hline HD $140283 \ldots \ldots \ldots \ldots$ & $0.007 \pm 0.011$ & $5753 \pm 61$ & $3.70 \pm 0.1^{\mathrm{b}}$ & -2.2 & 1.4 & 5.12 & 5.20 & $-2.25 \pm 0.07$ & 7.13 & 6.97 & $-1.68 \pm 0.03$ & $0.57 \pm 0.10$ \\
\hline 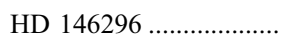 & $0.009 \pm 0.011$ & $5733 \pm 30$ & $4.13 \pm 0.11$ & -0.6 & 1.4 & 6.69 & 6.73 & $-0.72 \pm 0.12$ & 8.49 & 8.24 & $-0.41 \pm 0.03$ & $0.31 \pm 0.14$ \\
\hline 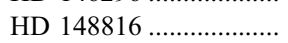 & $0.002 \pm 0.002$ & $5825 \pm 38$ & $4.14 \pm 0.1^{\mathrm{b}}$ & -0.6 & 1.5 & 6.68 & 6.71 & $-0.74 \pm 0.11$ & 8.67 & 8.38 & $-0.27 \pm 0.02$ & $0.47 \pm 0.12$ \\
\hline HD $160617 \ldots \ldots \ldots$ & $0.022 \pm 0.005$ & $6065 \pm 37$ & $3.82 \pm 0.10$ & -1.6 & 1.8 & 5.70 & 5.75 & $-1.70 \pm 0.08$ & 7.43 & 7.25 & $-1.40 \pm 0.05$ & $0.30 \pm 0.10$ \\
\hline 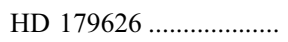 & $0.018 \pm 0.002$ & $5818 \pm 56$ & $3.85 \pm 0.11$ & -1.0 & 1.5 & 6.28 & 6.33 & $-1.12 \pm 0.10$ & 8.40 & 8.13 & $-0.52 \pm 0.05$ & $0.60 \pm 0.13$ \\
\hline 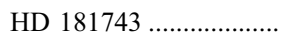 & $0.007 \pm 0.007$ & $6021 \pm 80$ & $4.45 \pm 0.11$ & -1.6 & 1.5 & 5.64 & 5.70 & $-1.75 \pm 0.08$ & 7.65 & 7.47 & $-1.18 \pm 0.02$ & $0.57 \pm 0.11$ \\
\hline HD $188031 \ldots \ldots \ldots \ldots \ldots \ldots$ & $0.016 \pm 0.016$ & $6196 \pm 63$ & $4.13 \pm 0.30$ & -1.5 & 1.5 & 5.77 & 5.81 & $-1.64 \pm 0.06$ & 7.77 & 7.55 & $-1.10 \pm 0.04$ & $0.54 \pm 0.11$ \\
\hline HD $193901 \ldots \ldots \ldots \ldots \ldots$ & $0.002 \pm 0.002$ & $5721 \pm 38$ & $4.52 \pm 0.1^{\mathrm{b}}$ & -0.9 & 1.2 & 6.41 & 6.46 & $-0.99 \pm 0.09$ & 8.21 & 8.01 & $-0.64 \pm 0.02$ & $0.35 \pm 0.11$ \\
\hline HD $194598 \ldots \ldots \ldots \ldots$ & $0.000 \pm 0.000$ & $5970 \pm 56$ & $4.31 \pm 0.1^{\mathrm{b}}$ & -0.9 & 1.5 & 6.32 & 6.37 & $-1.08 \pm 0.07$ & 8.22 & 7.99 & $-0.66 \pm 0.05$ & $0.42 \pm 0.11$ \\
\hline HD $215801 \ldots \ldots \ldots \ldots \ldots$ & $0.001 \pm 0.001$ & $6085 \pm 34$ & $3.83 \pm 0.15$ & -2.0 & 1.5 & 5.23 & 5.29 & $-2.16 \pm 0.08$ & 7.28 & 7.10 & $-1.55 \pm 0.04$ & $0.61 \pm 0.10$ \\
\hline HD $340279 \ldots \ldots \ldots$ & $0.020 \pm 0.012$ & $6521 \pm 102$ & $4.31 \pm 0.15$ & -2.3 & 1.5 & 4.94 & 4.97 & $-2.48 \pm 0.08$ & 6.69 & 6.49 & $-2.16 \pm 0.12$ & $0.32 \pm 0.17$ \\
\hline LP0815-43 .................... & $0.024 \pm 0.015$ & $6622 \pm 55$ & $4.28 \pm 0.15$ & -2.4 & 1.5 & 4.85 & 4.87 & $-2.58 \pm 0.06$ & 6.58 & 6.34 & $-2.31 \pm 0.21$ & $0.27 \pm 0.23$ \\
\hline
\end{tabular}

Note.-We adopt $A(\mathrm{Fe})_{\odot}=7.45$ and $A(\mathrm{O})_{\odot}=8.65$.

a The error given here is only the line-to-line scatter.
b A minimum error of 0.1 dex was has been adopted; the calculated error is smaller. 
11 colors for each sample star $(\S 5)$, alleviating in this way the impact of photometric errors.

\subsection{Extinction from Strömgren Photometry}

N04 determined interstellar reddening for the sample stars employing Strömgren $u v b y-\beta$ photometry and the calibration by Schuster \& Nissen (1989), including a zero-point correction of +0.005 mag (Nissen 1994).

The $E_{b-y}$ given by N04 was transformed to $E_{B-V}$ by applying $E_{B-V}^{u v b y-\beta}=1.35 E_{b-y}$ (Crawford 1975). When the $E_{b-y}$ value given by N04 was negative we adopted $E_{B-V}^{u v b y-\beta}=0.0$.

\subsection{Comparisons and Adopted Reddening}

In Figure 2 are compared the different $E_{B-V}$ values determined above. There are not clear correlations between the different methods. Fortunately, most of the points $\left(0.005<E_{B-V}<\right.$ 0.030 ) are randomly distributed along the $1: 1$ line, which means that a correlation may exists, but it is hidden by the errors in the reddening determinations. A larger sample, including very reddened stars (at least up to $E_{B-V}=0.1 \mathrm{mag}$ ) is necessary in order to evaluate the different methods employed to estimate the reddening.

The mean differences between the methods are the following: $E_{B-V}^{\mathrm{maps}}-E_{B-V}^{\mathrm{SED}}=0.002 \mathrm{mag}(\sigma=0.011 \mathrm{mag}) ; E_{B-V}^{\mathrm{maps}}-E_{B-V}^{u v b y-\beta}=$ $0.004 \mathrm{mag}(\sigma=0.012 \mathrm{mag}) ; E_{B-V}^{u v b y-\beta}-E_{B-V}^{\mathrm{SED}}=0.000 \mathrm{mag}$ ( $\sigma=0.015 \mathrm{mag}$ ). Considering that there are not significant zeropoint differences between the different methods, we adopted the mean value:

$$
E_{B-V}^{\text {adopted }}=\left(E_{B-V}^{\text {maps }}+E_{B-V}^{\mathrm{SED}}+E_{B-V}^{u v b y-\beta}\right) / 3
$$

In Table 4 are shown the adopted $E_{B-V}$ values and the scatter $\sigma$ between the three methods (typically $\sigma=0.008 \mathrm{mag}$ ).

\section{EFFECTIVE TEMPERATURES}

We used the new IRFM temperature scale of RM05b, which is based on a homogeneous analysis of more than $10^{3}$ stars, for which IRFM temperatures were obtained employing updated atmospheric parameters (RM05a). The main improvements compared with previous works are a better coverage of the atmospheric parameters space $\left(T_{\mathrm{eff}}, \log g,[\mathrm{Fe} / \mathrm{H}]\right)$, the use of up-to-date metallicities, and the fit of trends in the residuals, thus eliminating any spurious trend in the $T_{\text {eff }}$ :color: $[\mathrm{Fe} / \mathrm{H}]$ relations (RM05b). The use of updated metallicities and the good coverage of the atmospheric parameters space were crucial to derive reliable $T_{\text {eff }}$ calibrations, greatly helping to distinguish noise from real trends with metallicity. Seventeen colors were calibrated in the UBV, uvby, Vilnius, Geneva, RI (Cousins), DDO, Tycho (Hipparcos), and Two Micron All Sky Survey (2MASS) photometric systems (RM05b).

The colors of the sample stars were mainly obtained from the General Catalogue of Photometric Data (Mermilliod et al. 1997), the Hipparcos and Tycho Catalogue (ESA 1997), and the final release of $2 \mathrm{MASS}^{5}$ Almost all stars in the sample have $B-V, b-y$, Geneva, and 2MASS colors, and, when available, we also used Vilnius, Cousins, DDO, and Tycho colors. At least four colors were used for the temperature determination, although for most stars 11 colors were available. The mean, weighted average (using the error of each color calibration as

\footnotetext{
${ }^{5}$ See R. M. Cutri et al. 2003, VizieR Online Data Catalog, II/246.
}

weight), and trimean ${ }^{6}$ temperatures were computed, as well as the standard deviation, weighted error and quartile deviation. In general all estimates agree very well, except when an outlier was present. We adopted the trimean and the pseudo-standard (quartile) deviation, which is the robust equivalent of $\sigma$ in a normal distribution. ${ }^{7}$ The adopted temperatures and $\sigma$ are shown in Table 4.

\section{GRAVITIES}

Good $[\pi / \sigma(\pi)>4.5]$ Hipparcos parallaxes were employed to obtain trigonometric surface gravities [ $\log g($ Hip $)]$ with errors (only due to $\pi$ ) lower than 0.2 dex in $\log g$. The stellar mass was obtained from enhanced $\alpha$-element $Y^{2}$ isochrones (Kim et al. 2002; Demarque et al. 2004), by finding the closest isochrone to the $T_{\text {eff }}($ IRFM $) /$ luminosity (Hipparcos) $/[\mathrm{Fe} / \mathrm{H}](\mathrm{Fe}$ II) of the sample star. In this way we simultaneously determined isochronal ages and masses. Typical ages and masses are about $11 \mathrm{Gyr}$ and $0.82 M_{\odot}$, respectively. The use of other isochrones (e.g., Padova or Victoria) result in similar masses $\left(\approx 0.8 M_{\odot}\right.$ for a 12 Gyr turnoff at $[\mathrm{Fe} / \mathrm{H}]=-2.3)$, but we have adopted the $Y^{2}$ isochrones because they extend to $[\mathrm{Fe} / \mathrm{H}]=-3.3$, while most Padova and Victoria isochrones are available only for $[\mathrm{Fe} / \mathrm{H}] \gtrsim-2.3$.

We also estimated surface gravities from $Y^{2}$ isochrones $\left[\log g\left(Y^{2}\right)\right]$. For turnoff stars only one solution is found at a given $T_{\text {eff }}$, but for other stars the solution is twofold. When good parallaxes were available the solution closer to $\log g$ (Hip) was chosen, otherwise we used as additional constraints (1) photometric $M_{V}$ as determined from Strömgren photometry by N04, (2) Hipparcos parallaxes [when $\pi / \sigma(\pi)>2$ ], and (3) previous log $g$ data given in the literature. In some cases even when all these constraints were used it was still unclear (within the errors) whether the star was below or above the turnoff. In those cases we adopted as a compromise $\log g\left(Y^{2}\right)=\log g$ (turnoff), and due to this uncertainty in evolutionary status the error in $\log g\left(Y^{2}\right)$ for those stars is about 0.25 dex (this is the maximum possible $\log g$ shift from the turnoff to both the main-sequence and the subgiant branch).

For about half of the sample we have available good Hipparcos parallaxes; hence we can check our isochronal gravities. As shown in Figure 3, both agree very well, with a mean difference $\log g\left(Y^{2}\right)-\log g($ Hip $)=-0.015(\sigma=0.090)$. Based on this comparison, we adopted an error of 0.15 dex for $\log g\left(Y^{2}\right)$, except when there was a large uncertainty in evolutionary status, in which case an error of 0.3 dex was assigned.

The adopted surface gravity is the weighted average of $\log g\left(Y^{2}\right)$ and $\log g($ Hip $)$, and the adopted error is $\sigma_{\log g}=$ $\max \left(0.1 \mathrm{dex}, \sigma, \sigma_{\text {weighted }}\right)$. These surface gravities and $1 \sigma$ errors are given in Table 4.

\section{ABUNDANCES}

We employed Kurucz overshooting model atmospheres ${ }^{8}$ with 72 layers (Castelli et al. 1997), adopting a metallicity [M/H] (Table 4) about 0.2 dex higher than the iron abundance $[\mathrm{Fe} / \mathrm{H}]$ obtained in the literature (including the results obtained in this work), in order to compensate for the enhancement of $\alpha$-elements in halo stars (e.g., Sneden et al. 1994; Fulbright \& Kraft 1999). The use of solar-scaled Kurucz models instead of $\alpha$-enhanced

\footnotetext{
6 The trimean $T$ is a robust estimate of central tendency. We adopted Tukey's trimean $T=(\mathrm{Q} 1+2 \times$ median $+\mathrm{Q} 3) / 4$, where $\mathrm{Q} 1$ and $\mathrm{Q} 3$ are the first and third quartile.

7 The pseudo-standard deviation $\sigma$ was obtained from the quartile deviation $\mathrm{QD}[=(\mathrm{Q} 3-\mathrm{Q} 1) / 2]$, employing $\sigma=3 / 2 \mathrm{QD}$.

8 See http://kurucz.harvard.edu.
} 


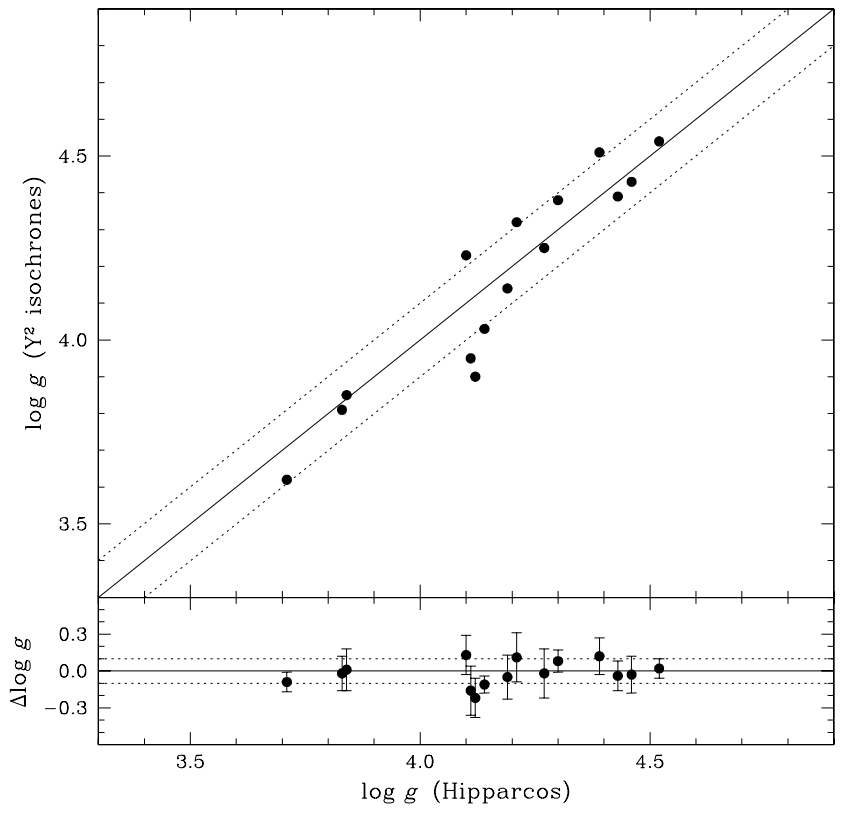

FIG. 3.-Comparison of surface gravities determined from Hipparcos parallaxes and $Y^{2}$ isochrones. The error bars include $1 \sigma$ errors in Hipparcos parallaxes and errors in mass and temperature. Solid and dotted lines correspond to the $1: 1$ line and \pm 0.1 dex uncertainties, respectively.

models have negligible impact on the $[\mathrm{O} / \mathrm{Fe}]$ ratio derived in the present work (see $\S 7.1$ and Table 5, where we show the sensitivity of $[\mathrm{O} / \mathrm{Fe}]$ to $[\mathrm{M} / \mathrm{H}])$.

The calculations were performed in LTE and NLTE employing the code NATAJA, which is described in Shchukina \& Trujillo
Bueno (2001) and Shchukina et al. (2005). The adopted atomic data for oxygen and iron were described in $\S 3$. LTE computations were also done with the latest version of MOOG (Sneden 1973). Both LTE computations agree very well, typically within 0.015 and 0.025 dex for Fe II and $\mathrm{O}$ I, respectively. The small differences are probably due to different continuum opacities adopted in the codes.

For stars with $[\mathrm{Fe} / \mathrm{H}] \geq-2$ we determined microturbulence velocities $v_{t}$ by requiring no dependence of $[\mathrm{Fe} / \mathrm{H}]$ against reduced equivalent width; we found a typical $v_{t}=1.5 \mathrm{~km} \mathrm{~s}^{-1}$. For stars with $[\mathrm{Fe} / \mathrm{H}]<-2$ the $\mathrm{Fe}$ II lines and $\mathrm{O}_{\text {I }}$ triplet are very weak and essentially independent of microturbulence (Table 5). For these very metal-poor stars we adopted $v_{t}=1.5 \mathrm{~km} \mathrm{~s}^{-1}$ (N04).

In Table 4 are given the oxygen and iron abundances derived in the present work, as well as the line-to-line scatter (i.e., errors mainly due to errors in $W_{\lambda}$ and $g f$-values). The $[\mathrm{O} \text { I/Fe II }]_{\mathrm{NLTE}}$ ratios obtained in this work are plotted in Figures $4 a-4 c$, as a function of $[\mathrm{Fe} / \mathrm{H}], T_{\text {eff }}$ and $\log g$, respectively.

In this work we obtained a mean $[\mathrm{O} / \mathrm{Fe}]=0.49 \mathrm{dex}$ (average) and a weighted mean $[\mathrm{O} / \mathrm{Fe}]=0.50 \mathrm{dex}($ see $\S 7.2)$.

\subsection{Overshooting versus No Overshooting}

We have assessed the effect of using the latest no convective overshooting (NOVER) Kurucz models (Castelli \& Kurucz 2003) instead of the overshooting models (Castelli et al. 1997) adopted in the present work.

The new NOVER Kurucz models adopt new ODFs, and models with both solar-scaled and $\alpha$-enhanced abundances are available in the range $-2.5 \leq[\mathrm{Fe} / \mathrm{H}] \leq+0.5$.

The solar-scaled NOVER models with a metallicity $[\mathrm{M} / \mathrm{H}]=$ $[\mathrm{Fe} / \mathrm{H}]+0.2$ dex resulted in essentially the same (within $0.01 \mathrm{dex}$ )

TABLE 5

Sensitivity to $T_{\text {eff }}, \log g, v_{t}$ AND $[\mathrm{M} / \mathrm{H}]$

\begin{tabular}{|c|c|c|c|c|}
\hline Abundance & $\Delta T_{\text {eff }}(+50 \mathrm{~K})$ & $\Delta \log g(+0.15$ dex $)$ & $\Delta v_{t}\left(+0.3 \mathrm{~km} \mathrm{~s}^{-1}\right)$ & $\Delta[\mathrm{M} / \mathrm{H}](+0.2 \mathrm{dex})$ \\
\hline \multicolumn{5}{|c|}{$\mathrm{BD}-133442(6510 / 4.10 /-2.53)^{\mathrm{a}}$} \\
\hline$[\mathrm{Fe}$ II $/ \mathrm{H}] \ldots \ldots \ldots \ldots \ldots$ & +0.01 & +0.05 & -0.01 & 0.00 \\
\hline$[\mathrm{O}$ I/H] & -0.03 & +0.06 & 0.00 & 0.00 \\
\hline 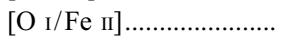 & -0.04 & +0.01 & +0.01 & 0.00 \\
\hline \multicolumn{5}{|c|}{$\mathrm{BD}+023375(6045 / 4.18 /-2.13)^{\mathrm{a}}$} \\
\hline 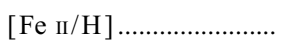 & +0.01 & +0.05 & -0.01 & 0.01 \\
\hline$[\mathrm{O}$ I/H $] \ldots \ldots \ldots \ldots \ldots \ldots \ldots$ & -0.03 & +0.05 & 0.00 & -0.01 \\
\hline 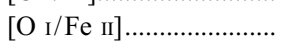 & -0.04 & +0.00 & +0.01 & -0.02 \\
\hline \multicolumn{5}{|c|}{ HD $140283(5753 / 3.70 /-2.25)^{\mathrm{a}}$} \\
\hline$[\mathrm{Fe}$ II $/ \mathrm{H}] \ldots \ldots \ldots \ldots \ldots \ldots$ & +0.01 & +0.05 & -0.02 & 0.00 \\
\hline$[\mathrm{O}$ I $/ \mathrm{H}] \ldots \ldots \ldots \ldots \ldots \ldots \ldots$ & -0.03 & +0.05 & 0.00 & 0.00 \\
\hline$[\mathrm{O}$ I $/ \mathrm{Fe}$ II $] \ldots \ldots \ldots \ldots \ldots \ldots \ldots$. & -0.04 & 0.00 & +0.02 & 0.00 \\
\hline \multicolumn{5}{|c|}{ HD $160617(6065 / 3.82 /-1.70)^{\mathrm{a}}$} \\
\hline 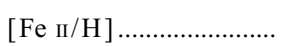 & +0.00 & +0.04 & -0.03 & 0.00 \\
\hline$[\mathrm{O}$ I/H] $] \ldots \ldots \ldots \ldots \ldots \ldots$ & -0.03 & +0.04 & -0.01 & -0.01 \\
\hline$\left[\mathrm{O}_{\mathrm{I}} / \mathrm{Fe}\right.$ II $] \ldots \ldots \ldots \ldots \ldots \ldots . . . . . . . .$. & -0.03 & 0.00 & +0.02 & -0.01 \\
\hline \multicolumn{5}{|c|}{ G53-41 $(5970 / 4.38 /-1.16)^{\mathrm{a}}$} \\
\hline$[\mathrm{Fe}$ II $/ \mathrm{H}] \ldots \ldots \ldots \ldots \ldots \ldots$ & 0.00 & +0.05 & -0.05 & +0.01 \\
\hline$[\mathrm{O}$ I/H] & -0.04 & +0.04 & -0.01 & -0.01 \\
\hline$[\mathrm{O}$ I/Fe II] $] \ldots \ldots \ldots . . . . . . . . . . .$. & -0.04 & -0.01 & +0.04 & -0.02 \\
\hline
\end{tabular}

a $T_{\text {eff }} / \log g /[\mathrm{Fe} / \mathrm{H}]$. 

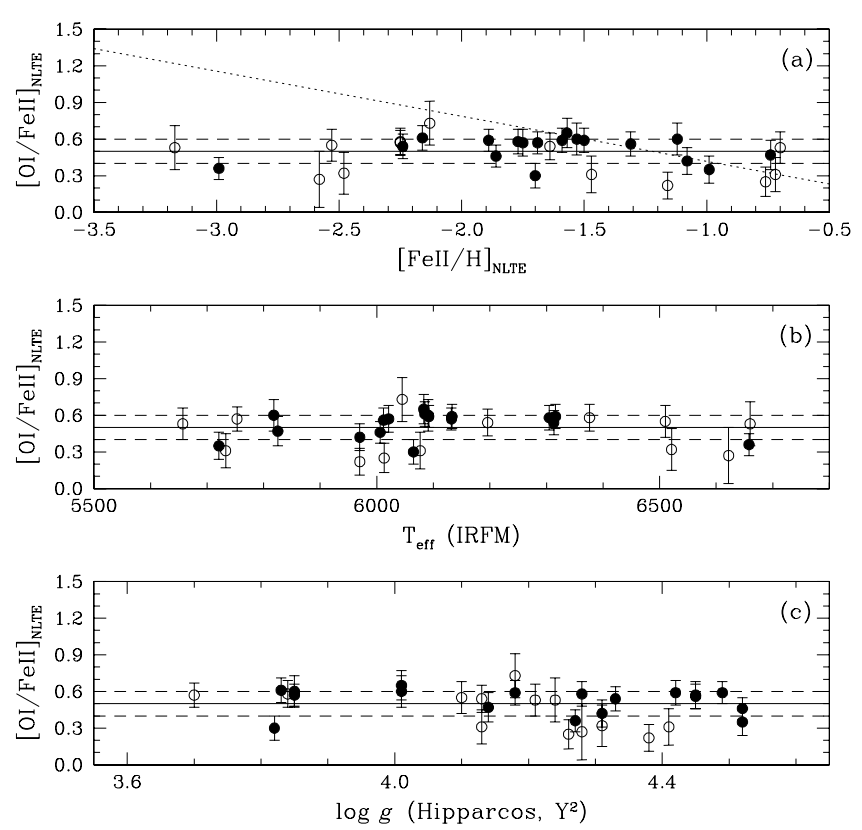

FIG. 4.-Oxygen-to-iron ratios vs. (a) iron abundance, $(b) T_{\text {eff, }}$ and (c) $\log g$. Filled and open circles represent stars with uncertainties lower and higher than $\Delta E_{B-V}=0.01 \mathrm{mag}$, respectively. The mean $[\mathrm{O} / \mathrm{Fe}]=+0.5$ is shown by solid lines, and \pm 0.1 dex uncertainties are shown by dashed lines. A linear relation between $[\mathrm{O} / \mathrm{Fe}]$ and $[\mathrm{Fe} / \mathrm{H}]$ previously found in other studies of the $\mathrm{O}_{\text {I }}$ triplet (Mishenina et al. 2000; Israelian et al. 1998, 2001; Boesgaard et al. 1999) is shown by a dotted line with a slope $=-0.35$ (top panel). [See the electronic edition of the Journal for a color version of this figure.]

oxygen and iron abundances as those obtained with the $\alpha$-enhanced NOVER models, which supports our choice of $[\mathrm{M} / \mathrm{H}]$ for the model atmospheres.

The use of the new NOVER models (both solar-scaled and $\alpha$-enhanced) gives lower abundances compared to overshooting models. The solar oxygen and iron abundances obtained from $\mathrm{Fe}$ II and $\mathrm{O}$ I lines are both reduced by 0.06 dex with the new NOVER models, while for the sample stars the Fe and $\mathrm{O}$ abundances are about $0.06-0.07$ and $0.08-0.10$ dex lower, respectively. Therefore, the use of the latest NOVER Kurucz models does not affect the results presented here, since the $[\mathrm{Fe} / \mathrm{H}]$, $[\mathrm{O} / \mathrm{H}]$, and $[\mathrm{O} / \mathrm{Fe}]$ ratios derived with the NOVER models are roughly preserved. Indeed, there is a small reduction of about 0.02 dex in $[\mathrm{O} / \mathrm{Fe}]$ when the new Kurucz models without convective overshooting are employed, reinforcing thus the low $[\mathrm{O} / \mathrm{Fe}]$ ratios found in the present work.

\subsection{Intrinsic Scatter and Errors}

We found a moderate star-to-star scatter in $[\mathrm{O} / \mathrm{Fe}]$ of $\sigma_{\mathrm{obs}}=$ 0.136 dex. The two main sources of observed star-to-star scatter are reddening and statistical errors (line-to-line scatter). The error in reddening introduces error in the temperature (and to some extent also in $\log g$ ), thus affecting the oxygen (and iron) abundance. An uncertainty of $0.01 \mathrm{mag}$ in $E_{B-V}$ corresponds to an uncertainty of $\pm 52 \mathrm{~K}$ in $T_{\text {eff. }}$.

In Figure 4 it is shown that the stars with an uncertainty $\Delta E_{B-V}>0.01 \mathrm{mag}$ (open circles) are the ones that deviate the most from the mean $[\mathrm{O} / \mathrm{Fe}]$. In fact, while $54 \%$ of the sample stars with $\Delta E_{B-V}$ mag (Fig. 4, open circles) deviate by more than 0.1 dex from $[\mathrm{O} / \mathrm{Fe}]=+0.5 \mathrm{dex}$, only $28 \%$ of the sample stars with lower reddening uncertainties (Fig. 4, filled circles) show the same discrepant behavior. Considering only the stars with $\Delta E_{B-V} \leq 0.01 \mathrm{mag}$, the observed scatter in $[\mathrm{O} / \mathrm{Fe}]$ is reduced to $\sigma=0.10$ dex.
Other sources of errors are due to uncertainties in the parameters of the model atmosphere $\left(T_{\text {eff }}, \log g, v_{t},[\mathrm{M} / \mathrm{H}]\right)$. In Table 5 are shown the impact of errors for changes of $\Delta T_{\text {eff }}=50 \mathrm{~K}$, $\Delta \log g=0.15 \mathrm{dex}, \Delta v_{\mathrm{t}}=0.3 \mathrm{~km} \mathrm{~s}^{-1}$, and $\Delta[\mathrm{M} / \mathrm{H}]=0.2 \mathrm{dex}$.

For each star in the sample we estimated the error in $[\mathrm{O} / \mathrm{Fe}]$ considering (1) the uncertainty in reddening given in column (2) of Table 4, adopting an error of 0.04 dex per $0.01 \mathrm{mag}$, due to its impact on $T_{\text {eff }}$; (2) the uncertainties in $T_{\text {eff }}$ and $\log g$ given in columns (3) and (4) of Table 4; (3) an error of $0.3 \mathrm{~km} \mathrm{~s}^{-1}$ in microturbulence; and (4) uncertainty of $0.2 \mathrm{dex}$ in $[\mathrm{M} / \mathrm{H}]$ of the model atmosphere; and (5) statistical uncertainties (line-to-line scatter) in the abundances of iron and oxygen given in columns (9) and (12) of Table 4, respectively. The error in $[\mathrm{O} / \mathrm{Fe}]$ is shown in the last column of Table 4. The weighted mean (adopting the inverse square of the errors as weights) of the oxygen-to-iron ratio is $[\mathrm{O} / \mathrm{Fe}]=+0.50 \mathrm{dex}$.

The mean error in $[\mathrm{O} / \mathrm{Fe}]$ is $0.121 \mathrm{dex}($ trimean $=0.112 \mathrm{dex})$, which is 0.015 dex lower than the observed star-to-star scatter in $[\mathrm{O} / \mathrm{Fe}]\left(\sigma_{\mathrm{obs}}=0.136 \mathrm{dex}\right)$. This means that the errors were slightly underestimated, or that there is some small intrinsic scatter in the sample.

In addition to the errors reported in Table 4, there may be systematic errors in our analysis due to errors in the IRFM $T_{\text {eff }}$ scale, NLTE effects, and granulation effects (e.g., Asplund 2006).

\section{DISCUSSION}

Since the equivalent widths used in this work were taken from Ake04 and N04, we first compare our results to theirs ( $§ 8.1$ ); then we discuss previous studies, especially those works claiming a step increase in $[\mathrm{O} / \mathrm{Fe}]$ for lower metallicities based on the $\mathrm{O}_{\mathrm{I}}$ triplet $(\S 8.2)$ and UV OH lines $(\S 8.3)$. We also compare our results with previous works that obtained a flat $[\mathrm{O} / \mathrm{Fe}]$ based on hot $T_{\text {eff }}$ scales $(\S 8.4)$. Then, we discuss whether high effective temperatures for metal-poor turnoff stars are physically reasonable, as well as the $T_{\text {eff }}$ of hyper-metal-poor turnoff stars $(\S 8.5)$.

\subsection{Comparison with Ake04/N04}

The main differences between our work and that of Ake04/ N04 are a different $T_{\text {eff }}$ scale (and reddening), a new set of $g f$ values for Fe II, NLTE calculations for both Fe II and O I, and the use of different model atmospheres. Note that the NLTE corrections $\delta_{\mathrm{NLTE}}\left(\equiv A^{\mathrm{NLTE}}-A^{\mathrm{LTE}}\right)$ for $\mathrm{O}$ I used by Ake04 were interpolated from previous calculations by Nissen et al. (2002), while in our case we explicitly computed the LTE and NLTE abundances for each star.

In Figure 5 are shown the differences between the present work and that of Ake04/N04, as a function of [Fe/H] (left panels) and $T_{\text {eff }}$ (right panels). In Figures $5 b, 5 c, 5 f$, and $5 g$ an outlier can be seen at $[\mathrm{Fe} / \mathrm{H}] \approx-0.7$ and $T_{\text {eff }} \approx 5650 \mathrm{~K}$, respectively. This is probably due to a typo in the oxygen abundance of $\mathrm{BD}$ +08 3065 (G016-013) given by Ake04, since its $\mathrm{O}$ abundance cannot be lower than that of HD 146296, which has both lower $W_{\lambda}$ and higher $T_{\text {eff }}$ than $\mathrm{BD}+083065$.

The differences $\Delta[\mathrm{Fe} / \mathrm{H}]$ and $\Delta A_{\mathrm{Fe}}^{\mathrm{LTE}}$ are shown in Figures $5 a$ and $5 e$ with filled and open circles, respectively. Despite our higher $T_{\text {eff }}$ (as shown in Figs. $5 d$ and $5 h$ ), our $A_{F e}^{\mathrm{LTE}}$ abundances are in excellent agreement with Ake04/N04. This can be explained by the low sensitivity of the iron abundance to changes in $T_{\text {eff }}$ (Table 5). The difference in $[\mathrm{Fe} / \mathrm{H}]$ is independent of $[\mathrm{Fe} / \mathrm{H}]$ and $T_{\text {eff }}$, but with an offset of $0.11 \mathrm{dex}$, which is due to the use of different model atmospheres, solar abundances, ${ }^{9} g f$-values and

\footnotetext{
9 The solar Fe abundance was not determined by Ake04/N04, but it should be in the same system of Nissen et al. (2002), where $A(\mathrm{Fe})_{\odot}=7.53$ was found.
} 


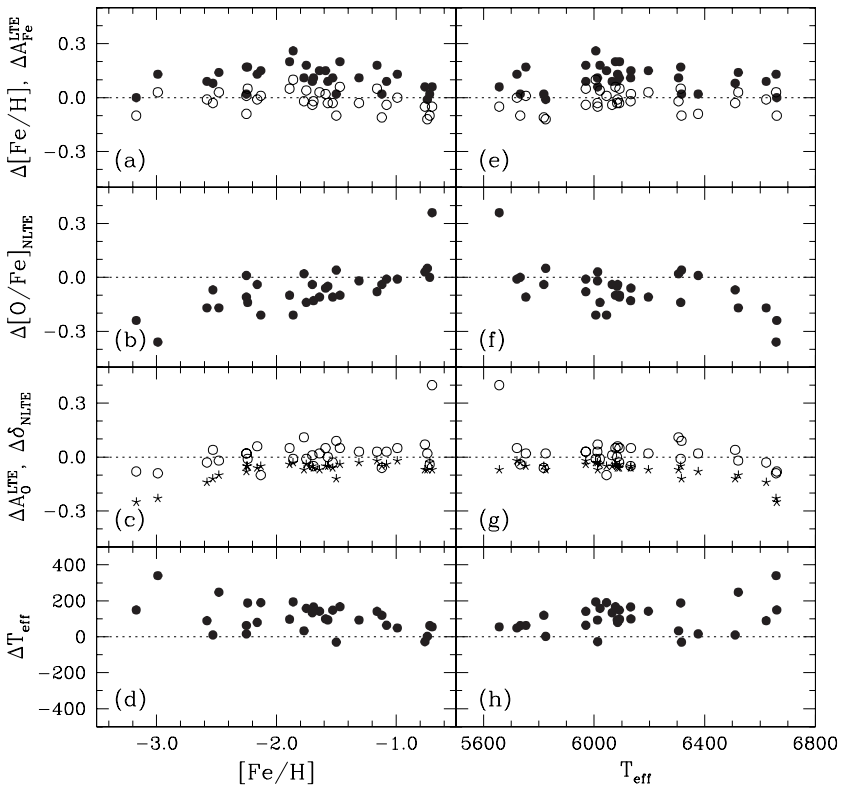

FIG. 5.-Differences between the present work and Ake04 are shown in the left and right panels as a function of $[\mathrm{Fe} / \mathrm{H}]$ and $T_{\text {eff }}$, respectively: $\Delta[\mathrm{Fe} / \mathrm{H}]$ ( filled circles); $\Delta A_{\mathrm{Fe}}^{\mathrm{LTE}}$ (open circles); $\Delta[\mathrm{O} / \mathrm{Fe}]_{\mathrm{NLTE}}\left(\right.$ filled circles); $\Delta A_{\mathrm{O}}^{\mathrm{LTE}}$ (open circles); $\Delta \delta_{\mathrm{NLTE}}$ (stars); $\Delta T_{\mathrm{eff}}($ filled circles $)$.

NLTE corrections [Ake04/N04 do not correct for NLTE effects, while our $\delta_{\mathrm{NLTE}}(\mathrm{Fe}$ II $\left.) \approx 0.046 \mathrm{dex}\right]$.

Our $[\mathrm{O} / \mathrm{Fe}]_{\mathrm{NLTE}}$ ratios are lower than those in Ake04, as can be seen in Figures $5 b$ and $5 f$. The difference $\Delta[\mathrm{O} / \mathrm{Fe}]_{\text {NLTE }}$ depends on metallicity, reaching about -0.3 dex at $[\mathrm{Fe} / \mathrm{H}]=-3$. This is partly explained by our higher $T_{\text {eff }}$ for lower metallicities (Figs. $5 d$ and $5 h$ ), but also for the small NLTE corrections used by Ake 04 for the most metal-poor stars. In Figures $5 c$ and $5 g$ the differences in LTE oxygen abundance $\left(\Delta A_{\mathrm{O}}^{\mathrm{LTE}}\right)$ and in NLTE corrections ( $\left.\Delta \delta_{\mathrm{NLTE}}\right)$ are represented by open circles and stars, respectively. There is a good agreement in the $A_{\mathrm{O}}^{\mathrm{LTE}}$, although for the most metal-poor stars we expected to see a larger discrepancy in $A_{\mathrm{O}}^{\mathrm{LTE}}$ due to the lower $T_{\text {eff }}$ adopted by Ake04. The NLTE corrections are similar for $[\mathrm{Fe} / \mathrm{H}]>-2.5$, with an small offset of -0.053 dex. For lower metallicities our NLTE corrections are considerably larger, being about 0.25 larger (more negative) at $[\mathrm{Fe} / \mathrm{H}] \approx-3$.

The interpolated $\delta_{\text {NLTE }}$ for the five stars with the lowest metallicities have been wrongly estimated by Ake04 (M. Asplund 2005, private communication). In fact, the original NLTE correction by Nissen et al. (2002) for LP 815-43, the star with the lowest metallicity $([\mathrm{Fe} / \mathrm{H}]=-2.7)$ in their sample, is $\delta_{\text {NLTE }}\left(\mathrm{O}_{\mathrm{I}}\right)=-0.25 \mathrm{dex}$, which is in excellent agreement with our $\delta_{\mathrm{NLTE}}\left(\mathrm{O}_{\mathrm{I}}\right)=-0.24 \mathrm{dex}$.

\subsection{Linear Relationship versus Flat $[\mathrm{O} / \mathrm{Fe}]$}

As can be seen in Figures $4 a-4 c$ the $[\mathrm{O} / \mathrm{Fe}]$ ratio is flat $([\mathrm{O} / \mathrm{Fe}] \approx+0.5)$ and independent of metallicity, temperature and surface gravity, respectively. On the other hand, some previous investigations of the $\mathrm{O}$ I triplet have found a steep linear relationship between $[\mathrm{O} / \mathrm{Fe}]$ and $[\mathrm{Fe} / \mathrm{H}]$ (Abia \& Rebolo 1989; Cavallo et al. 1997; Israelian et al. 1998, 2001; Mishenina et al. 2000 ), all with a similar slope of about -0.35 , and reaching $[\mathrm{O} / \mathrm{Fe}] \approx 1.1$ at $[\mathrm{Fe} / \mathrm{H}]=-3$. Interestingly, the work by Tomkin et al. (1992) found a flat $[\mathrm{O} / \mathrm{Fe}]$, but with a high mean $[\mathrm{O} / \mathrm{Fe}]=$ 0.8 . Note also that the analysis of the $\mathrm{O}$ i triplet by Nissen et al. (2002) shows only a mild dependence of [O/Fe] with metallicity, with $[\mathrm{O} / \mathrm{Fe}] \approx 0.4$ at $[\mathrm{Fe} / \mathrm{H}]=-1$, and increasing to
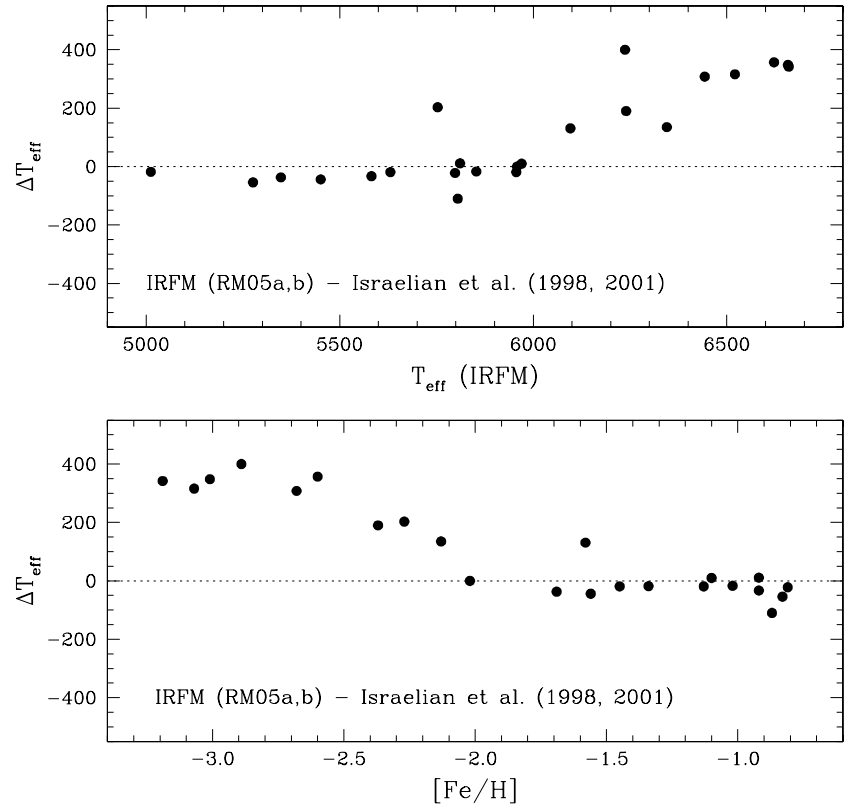

FIG. 6.- Temperatures obtained by us employing the IRFM $T_{\text {eff }}$ scale of RM05a and RM05b minus the temperatures from Israelian et al. $(1998,2001)$ as a function of $T_{\mathrm{eff}}$ (top panel) and $[\mathrm{Fe} / \mathrm{H}]$ (bottom panel).

$[\mathrm{O} / \mathrm{Fe}] \approx 0.6$ at $[\mathrm{Fe} / \mathrm{H}]=-2.5$. The $[\mathrm{O} / \mathrm{Fe}]$ ratios obtained by Ake04 are similar to those obtained by Nissen et al. (2002), showing a mild dependence with metallicity and reaching $[\mathrm{O} / \mathrm{Fe}] \approx 0.7$ at $[\mathrm{Fe} / \mathrm{H}]=-3$. However, as we have seen in $\S 8.1$, the mild slope found by Ake 04 can be reduced by employing our $T_{\text {eff }}$ scale and also proper NLTE corrections for $[\mathrm{Fe} / \mathrm{H}]<-2.5$.

For comparison purposes between our results and previous studies which have found a steep linear trend between $[\mathrm{O} / \mathrm{Fe}]$ and $[\mathrm{Fe} / \mathrm{H}]$, a typical linear relationship found by those works is shown with a dotted line in Figure $4 a$. Clearly, our results do not support previous claims for a large increase of $[\mathrm{O} / \mathrm{Fe}]$ for decreasing metallicities.

Besides the neglect (or underestimation) of NLTE effects for $\mathrm{O}$, the main reason for the large slope found in other works based on the $\mathrm{O}$ I triplet seems to be the lower $T_{\text {eff }}$ adopted. For example, we show in Figure 6 the difference $\Delta T_{\text {eff }}$ between the IRFM temperatures obtained with the $T_{\text {eff }}$ scale by RM05b and those adopted by Israelian et al. $(1998,2001)$. The difference $\Delta T_{\text {eff }}$ increases with decreasing $[\mathrm{Fe} / \mathrm{H}]$, reaching about $\Delta T_{\text {eff }}=$ $+350 \mathrm{~K}$ at $[\mathrm{Fe} / \mathrm{H}]=-3$, thus leading Israelian et al. (1998, $2001)$ to derive much higher (and metallicity-dependent) [O/Fe] ratios.

A better understanding of the differences between our temperatures and those adopted by Israelian et al. (1998, 2001) can be achieved by examining the differences between the temperature scales adopted by them and us (RM05b). Israelian et al. (1998) adopted the $b-y$ and $V-K T_{\text {eff }}$ calibration by Alonso et al. (1996), while Israelian et al. (2001) adopted $V-K$ calibrations by Alonso et al. (1996) and Carney (1983). In Figure 7 we show the differences between the $b-y$ and $V-K$ calibrations by RM05b and those by Alonso et al. (1996) and Carney (1983).

In their first paper, which mainly analyzes stars cooler than $6000 \mathrm{~K}$, Israelian et al. (1998) adopted the mean of the $b-y$ and $V-K$ calibrations by Alonso et al. (1996). As can be seen in Figure 7 , for $T_{\text {eff }}<6000 \mathrm{~K}$, the $b-y$ and $V-K$ Alonso et al. (1996) temperatures are higher and lower than RM05b, respectively. Thus the mean color temperatures are similar to RM05b, explaining the good agreement for $T_{\text {eff }}<6000 \mathrm{~K}$ (Fig. 6). 


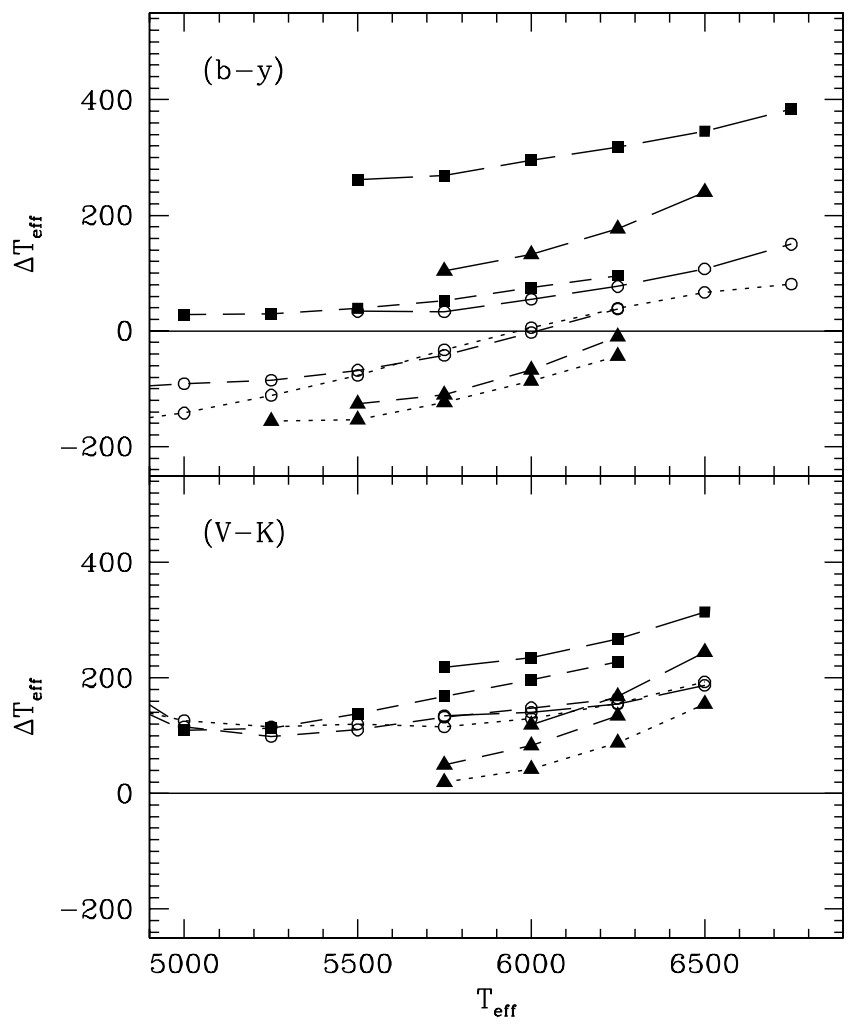

FIG. 7.-Top: Difference between the $(b-y)$ temperature scales by RM05b and Alonso et al. (1996; circles), Carney (1983; squares), and King (1993; triangles) at $[\mathrm{Fe} / \mathrm{H}]=-1$ (dotted lines), $[\mathrm{Fe} / \mathrm{H}]=-2$ (dashed lines), and $[\mathrm{Fe} / \mathrm{H}]=-3$ (long-dashed lines $)$. The solid line is at $\Delta T_{\text {eff }}=0$. Bottom: Same, but for $(V-K)$.

In their second paper, which deals mainly with very metalpoor $(-3.4<[\mathrm{Fe} / \mathrm{H}]<-2.5)$ stars hotter than $6000 \mathrm{~K}$, Israelian et al. (2001) adopted the mean from the $V-K$ calibrations by Alonso et al. (1996) and Carney (1983). Figure 7 shows that for $T_{\text {eff }}>6000 \mathrm{~K}$ the $V-K$ calibration by Alonso et al. is lower than that of RM 05 b by about $180 \mathrm{~K}$, while the $V-K$ calibration by Carney (1983) is lower than RM05b by about $340 \mathrm{~K}$ in the relevant $T_{\text {eff }}$ and [ Fe/H] range used by Israelian et al. (2001). So, on average, the calibrations used by Israelian et al. (2001) are about $250 \mathrm{~K}$ lower than those by RM05b. Besides, Israelian et al. (2001) neglected reddening corrections, which are important for distant early G/F dwarfs. For stars hotter than $6000 \mathrm{~K}$, we obtained a mean $E_{B-V}=0.016 \mathrm{mag}$, which is equivalent to $\Delta T_{\text {eff }} \approx 85 \mathrm{~K}$. So, considering altogether the neglect of reddening (85 K) and the use of the Alonso et al. (1996) and Carney (1983) calibrations $(250 \mathrm{~K})$, Israelian et al. (2001) temperatures are lower than RM05b by about $335 \mathrm{~K}$, which explain the large difference shown in Figure 6 for $T_{\text {eff }}>6000 \mathrm{~K}$.

A similar reasoning could be applied to explain the differences between the temperatures obtained from the calibrations by RM05b and those determined by other authors. We also show in Figure 7 the difference between our $T_{\text {eff }}$ scale and that of King (1993), which was used by Boesgaard et al. (1999), who also used the $T_{\text {eff }}$ scale by Carney (1983).

It is important to mention that not all works which have found large $[\mathrm{O} / \mathrm{Fe}]$ ratios are based entirely on photometric temperatures. For example, part of the sample analyzed by Mishenina et al. (2000) have temperatures determined from the fitting of $\mathrm{H} \alpha$ line profiles, and the other part was taken from the work by Cavallo et al. (1997), which is a mix of temperatures obtained from the excitation equilibrium of $\mathrm{Fe}_{\mathrm{I}}$ lines, photometric tem-

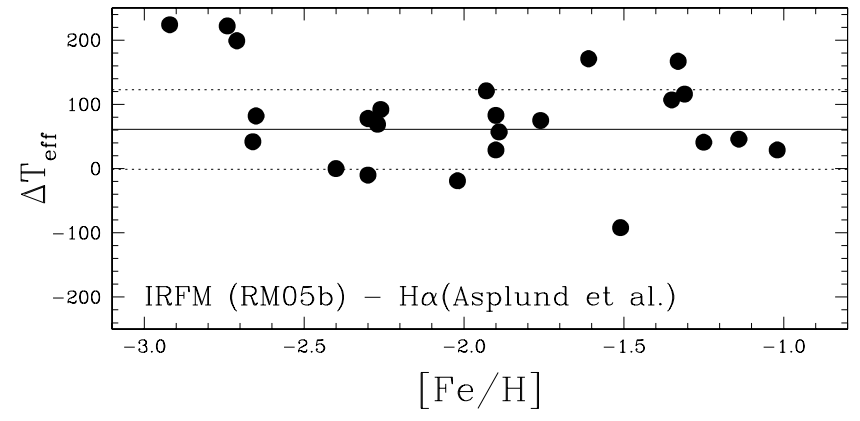

FIG. 8.-Temperatures employing the IRFM $T_{\text {eff }}$ scale by RM05b minus the $\mathrm{H} \alpha$ temperatures determined by Asplund et al. (2006). Excluding the three stars with $[\mathrm{Fe} / \mathrm{H}]<-2.7$, the zero-point shift is $61 \pm 62 \mathrm{~K}$, which is represented by a solid line. Dotted lines represent the scatter $(\sigma=62 \mathrm{~K})$.

peratures from the King (1993) $T_{\text {eff }}$ scale, temperatures from hydrogen profiles by Axer et al. (1994), or average $T_{\text {eff }}$ from several values reported in the literature.

In principle, the temperature from $\mathrm{H} \alpha$ should be reliable, but in practice the $\mathrm{H} \alpha$ temperatures are subject to problems in the continuum determination (wings falling in different orders), as well as to the treatment of convection (Castelli et al. 1997). For example, Castelli et al. (1997) found a $\mathrm{H} \alpha$ temperature of 6500 and $6700 \mathrm{~K}$ in Procyon, depending on the adopted prescription for convection. They obtained higher temperatures from other Balmer lines. Interestingly, Aufdenberg et al. (2005) have recently found that Kurucz convective overshooting model atmospheres may better represent the mean temperature structure of F stars similar to Procyon, since high-precision interferometric optical-red-infrared data of this star are consistent with a temperature structure with significant convective overshooting.

Although the absolute $\mathrm{H} \alpha$ temperatures may be in error, the relative temperatures should be reliable. In Figure 8 we show that the relative temperatures of metal-poor $(-3<[\mathrm{Fe} / \mathrm{H}]<-1)$ turnoff stars determined by Asplund et al. (2006) using an accurate modeling of the $\mathrm{H} \alpha$ line are in excellent agreement with those given by the IRFM $T_{\text {eff }}$ scale of RM05b, except for the three more metal-poor stars $([\mathrm{Fe} / \mathrm{H}]<-2.7)$, which have systematically lower $\mathrm{H} \alpha$ temperatures. Excluding those stars, the zeropoint difference (IRFM-H $\alpha$ ) is only $61 \pm 62 \mathrm{~K}$, similar to the difference $\Delta T_{\text {eff }}=34 \pm 95 \mathrm{~K}$ found by Asplund et al. (2006) between their $\mathrm{H} \alpha$ temperatures are those from the IRFM $T_{\text {eff }}$ scale of Alonso et al. (1996).

\subsection{The New IRFM $T_{\text {eff }}$ scale and Revised $[\mathrm{O} / \mathrm{Fe}]$ from the $U V \mathrm{OH}$ lines}

Fields et al. (2005) have recently used effective temperatures from Meléndez \& Ramírez (2004), which are based on the new IRFM $T_{\text {eff }}$ scale by RM05a and RM05b, in order to determine the oxygen abundance from the UV OH lines in 13 metal-poor dwarfs. Fields et al. (2005) find very high $[\mathrm{O} / \mathrm{Fe}]$ ratios, with a very steep slope: $[\mathrm{O} / \mathrm{Fe}]=-0.66[\mathrm{Fe} / \mathrm{H}]-0.25$, which is about twice as large as that claimed in previous works based on the UV OH lines (Israelian et al. 1998; Boesgaard et al. 1999). The slope is reduced when the single point with $[\mathrm{Fe} / \mathrm{H}]<-3$ and an extreme $[\mathrm{O} / \mathrm{Fe}] \approx+2.4$ is excluded, resulting in $[\mathrm{O} / \mathrm{Fe}]_{1 \mathrm{D}}^{\mathrm{UVH}}=$ $-0.30[\mathrm{Fe} / \mathrm{H}]+0.49$, which has a similar slope to that found by Israelian et al. (1998) and Boesgaard et al. (1999), but a higher constant term. Half of the higher constant term is explained by the different solar abundances adopted by Fields et al. (2005; K. A. Olive \& B. D. Fields 2005, private communication), leading to an increase of $+0.23 \mathrm{dex}$ in $[\mathrm{O} / \mathrm{Fe}]$. The other half is probably due to the use of a hotter $T_{\text {eff }}$ scale. 


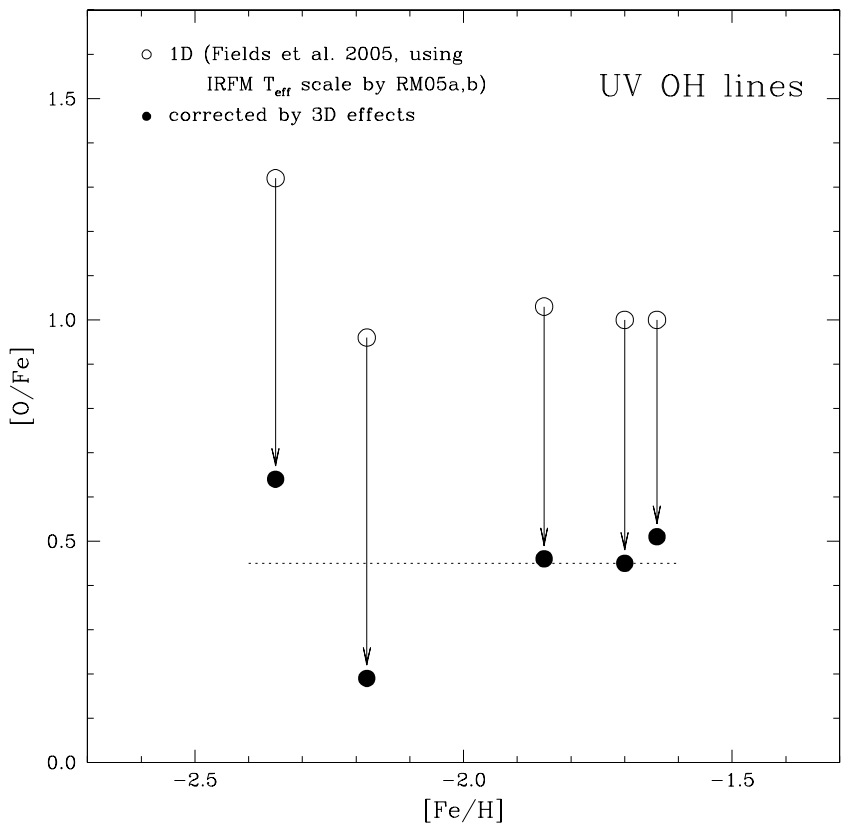

Fig. 9.- $[\mathrm{O} / \mathrm{Fe}]$ (from UV OH lines) vs. $[\mathrm{Fe} / \mathrm{H}]$ employing the new $T_{\text {eff }}$ scale by RM05a and RM05b. Open circles represent the $[\mathrm{O} / \mathrm{Fe}]$ ratios obtained in the one-dimensional analysis by Fields et al. (2005); filled circles represent the $[\mathrm{O} / \mathrm{Fe}]$ after three-dimensional effects (arrows) are taken into account (from threedimensional corrections computed by Asplund \& García Pérez 2001). The dotted line represents the mean three-dimension-corrected $[\mathrm{O} / \mathrm{Fe}]=+0.45 \mathrm{dex}$. [See the electronic edition of the Journal for a color version of this figure.]

As shown by Asplund \& García Pérez (2001), oxygen abundances from the UV OH lines may be severely overestimated by one-dimensional analyses, ${ }^{10}$ and the three-dimensional abundance corrections depend on metallicity and temperature. For example, for a star with $T_{\text {eff }} \approx 6000 \mathrm{~K}$, the three-dimensional correction $\Delta[\mathrm{O} / \mathrm{Fe}]_{3 \mathrm{D}-1 \mathrm{D}}^{\mathrm{UVOH}}$ increases from about -0.5 dex at $[\mathrm{Fe} / \mathrm{H}]=-2$, to -0.7 at $[\mathrm{Fe} / \mathrm{H}] \approx-3$. On the other hand, at $[\mathrm{Fe} / \mathrm{H}]=-3$, increasing the temperature from $T_{\mathrm{eff}} \approx 5890$ to $T_{\text {eff }} \approx 6200 \mathrm{~K}$ increases the $\Delta[\mathrm{O} / \mathrm{Fe}]_{3 \mathrm{D}-1 \mathrm{D}}^{\mathrm{UVH}}$ correction from -0.5 to -0.9 dex (Asplund \& García Pérez 2001). Therefore, the increase in the one-dimensional oxygen abundance from the UV $\mathrm{OH}$ lines due to higher temperatures is probably compensated by the increase in the three-dimensional corrections, resulting in low $[\mathrm{O} / \mathrm{Fe}]$ ratios.

The above reasoning is checked quantitatively in Figure 9, where we show the $[\mathrm{O} / \mathrm{Fe}]$ ratios obtained by Fields et al. (2005) using the temperatures by Meléndez \& Ramírez (2004), along with the corresponding $\Delta[\mathrm{O} / \mathrm{Fe}]_{3 \mathrm{D}-1 \mathrm{D}}^{\mathrm{UVOH}}$ corrections interpolated from three-dimensional calculations by Asplund \& García Pérez (2001). Since three-dimensional corrections are only available for stars with $T_{\text {eff }} \leq 6205 \mathrm{~K}$, we refrained from applying the corrections to the whole sample analyzed by Fields et al. (2005), but only to stars with $T_{\text {eff }}<6250 \mathrm{~K}$ (in fact, most stars shown in Fig. 9 have $T_{\text {eff }}<6150 \mathrm{~K}$ ). The mean corrected $[\mathrm{O} / \mathrm{Fe}]$ ratio is $[\mathrm{O} / \mathrm{Fe}]_{3 \mathrm{D}}^{\mathrm{UV} \mathrm{OH}}=0.45$. Although it is true that our $T_{\text {eff }}$ scale increases the $[\mathrm{O} / \mathrm{Fe}]$ ratio obtained from the $\mathrm{UV} \mathrm{OH}$ lines in a onedimensional analysis, it is also true that the three-dimensional corrections increase with higher $T_{\text {eff }}$ and lower $[\mathrm{Fe} / \mathrm{H}]$, thus compensating the first effect and resulting in a roughly flat (and low) $[\mathrm{O} / \mathrm{Fe}]$ ratio for halo stars (Fig. 9).

\footnotetext{
${ }^{10}$ Note that the large three-dimensional abundance corrections apply mainly to hot turnoff stars; therefore this is not necessarily in conflict with the recent low $[\mathrm{O} / \mathrm{Fe}]$ ratios found in the one-dimensional analysis of $\mathrm{UV}$ OH lines in subgiants by García Pérez et al. (2006).
}

It is important to mention that we have assumed above that the stellar parameters remain roughly unchanged with threedimensional model atmospheres. Shchukina et al. (2005) have recently performed $1.5 \mathrm{D}+\mathrm{NLTE}$ computations employing a threedimensional model atmosphere of the metal-poor subgiant HD 140283 , and based on spectroscopic constrains they suggested that the stellar parameters for this star may need modification when three-dimensional model atmospheres are employed. On the other hand, Asplund \& García Pérez (2001) show that when the effective temperatures of metal-poor stars of solar $T_{\text {eff }}$ are determined with the IRFM, only small changes in $T_{\text {eff }}$ (below $20 \mathrm{~K}$ ) are expected in three-dimensional analysis. The changes are even smaller (below $5 \mathrm{~K}$ ) for metal-poor turnoff stars. In their analysis of the UV OH lines, Fields et al. (2005) adopted effective temperatures by Meléndez \& Ramírez (2004), which are in the IRFM $T_{\text {eff }}$ scale of RM05b, and therefore those $T_{\text {eff }}$ should be little affected in three-dimensional model atmospheres. The main concern is probably the iron abundance, which for the only star (HD 140283) with a 1.5D+NLTE calculations in a threedimensional model atmosphere (Shchukina et al. 2005), is 0.25 dex higher than in the present one-dimensional analysis. The higher metallicity would result in a lower three-dimensional correction, which is compensated by the increase of $0.25 \mathrm{dex}$ in $[\mathrm{Fe} / \mathrm{H}]$, resulting in slightly lower $[\mathrm{O} / \mathrm{Fe}]$ ratios than those presented in Figure 9. Note also that the UV OH lines may be affected by NLTE effects, perhaps increasing the oxygen abundances from UV OH lines (Asplund \& García Pérez 2001). Full 3D+NLTE calculations of the UV OH lines are strongly encouraged.

It would be important to check whether $\mathrm{Fe}$ I and $\mathrm{Fe}$ II lines satisfy the excitation and ionization equilibrium in 3D+NLTE (and also 1D+NLTE), which may bring support (or not) to the new $T_{\text {eff }}$ scale by RM05b. Nevertheless, the NLTE calculations should be first checked employing stars with well-determined stellar parameters. This is a formidable theoretical and observational task, especially for very metal-poor turnoff stars, where high excitation potential $(3-5 \mathrm{eV}) \mathrm{Fe}$ I lines are extremely weak, yet important to minimize the degeneracy of stellar parameters based on spectroscopic equilibrium of iron lines. Besides, further work on establishing a reliable $g f$-scale of $\mathrm{Fe}$ I lines is required.

The determination of a reliable $A_{\mathrm{Fe}}^{\mathrm{NLTE}}$ is very important, since the oxygen-to-iron ratio depends on the adopted $\mathrm{Fe}$ abundance. In fact, as discussed in $\S 1$, the relatively low $[\mathrm{O} / \mathrm{Fe}]$ ratios found in the reanalysis of UV OH lines by King (2000) was partly due to large $\delta_{\text {NLTE }}(\mathrm{Fe}$ I) adopted from Thévenin \& Idiart (1999).

\subsection{Other Flat $[\mathrm{O} / \mathrm{Fe}]$ Ratios and Hot $T_{\text {eff }}$ Scales}

Our finding of a flat (and low) $[\mathrm{O} / \mathrm{Fe}]$ ratio using the permitted $\mathrm{O}$ I lines has been previously reported in the literature, although in a more restricted metallicity range.

This result has been achieved with different $T_{\text {eff }}$ scales, although it was first shown with the hot $T_{\text {eff }}$ scale by King (1993). Tomkin et al. (1992) obtained a flat $[\mathrm{O} / \mathrm{Fe}]$, but with a high mean $[\mathrm{O} / \mathrm{Fe}] \approx+0.8$. The reanalysis of Tomkin et al. data by King (2000), employing new stellar parameters and $A_{\mathrm{Fe}}^{\mathrm{NLTE}}$, resulted in a significant reduction of $[\mathrm{O} / \mathrm{Fe}]$, partly due to high $A_{\mathrm{Fe} I}^{\mathrm{NLTE}}$ abundances. Unfortunately, King (2000) only presented his $\mathrm{O}$ I reanalysis in plots, so a detailed comparison is not possible. The $[\mathrm{O} / \mathrm{Fe}]$ ratios obtained by both Tomkin et al. (1992) and King (2000) should be increased by about 0.2 dex due to the new low solar $\mathrm{O}$ and $\mathrm{Fe}$ abundances, increasing thus the discrepancy of Tomkin et al. with the present work. Note that the NLTE corrections adopted by Tomkin et al. (1992) are almost negligible, because they empirically included the effects of neutral $\mathrm{H}$ collisions, adjusting them so that the NLTE solar oxygen 
abundance from the $\mathrm{O}_{\mathrm{I}}$ triplet reproduced the high solar $A_{\mathrm{O}}$ obtained from the $\left[\mathrm{O}_{\mathrm{I}}\right]$ and infrared $\mathrm{OH}$ lines $\left(A_{\mathrm{O}}=8.92\right)$. In this way, Tomkin et al. significantly reduced the departures from LTE. However, recent studies (see $\S 3.1$ for references) show that the solar $\mathrm{O}$ abundance is much lower than previously thought. Our solar $A_{\mathrm{O}}^{\mathrm{NLTE}}$ from the $\mathrm{O}$ I triplet is in excellent agreement with the $\mathrm{O}$ abundance from other spectral features (see $\S 3.1$ ), hence the inclusion of neutral $\mathrm{H}$ collisions may not be necessary.

Primas et al. (2001) presented preliminary NLTE oxygen abundances from the $\mathrm{O}_{\mathrm{I}}$ triplet in a sample of dwarfs with $[\mathrm{Fe} / \mathrm{H}] \geq$ -2.4 . Their analysis employed three different sets of $T_{\text {eff: }}$ : the $(b-y)$ calibrations of Alonso et al. (1996) and Carney (1983), and temperatures from Thévenin \& Idiart (1999). Primas et al. $[\mathrm{O} / \mathrm{Fe}]$ ratios are roughly flat, with $[\mathrm{O} / \mathrm{Fe}] \approx+0.4$ for the Alonso et al. (1996) $T_{\text {eff }}$ scale, and $[\mathrm{O} / \mathrm{Fe}] \approx+0.5$ for the other two $T_{\text {eff }}$ scales. The preliminary results of Primas et al. are in good agreement with our work. This is not surprising, since the $(b-y)$ calibration of Alonso et al. (1996) employed by Primas et al. (2001) gives even hotter temperatures than those from the RM05b $T_{\text {eff }}$ scale, at least for $[\mathrm{Fe} / \mathrm{H}] \geq-2$ and $T_{\text {eff }}<6000 \mathrm{~K}$ (Fig. 7). On the other hand, the $(b-y)$ calibration of Carney (1983) at $[\mathrm{Fe} / \mathrm{H}]=-2$ is not much cooler (only $20-100 \mathrm{~K}$ lower) than the $(b-y)$ calibration of RM05b. It is unknown what $T_{\text {eff }}$ scale was employed by Thévenin \& Idiart (1999), since they refereed to a CDS catalog by Thévenin, where no details are given.

King (1993) found that the very large $[\mathrm{O} / \mathrm{Fe}]$ ratios derived by Abia \& Rebolo (1989) were partly due to large errors in equivalent widths (overestimated by about 24\%). Furthermore, as a solution to the discrepancy between the oxygen abundances obtained from the triplet and the forbidden lines, King (1993) suggested an increase of about $150-200 \mathrm{~K}$ in the $T_{\text {eff }}$ scale of dwarfs, finding in this way a flat $[\mathrm{O} / \mathrm{Fe}] \approx 0.5 \mathrm{dex}$ (his Fig. 9). On the other hand, Carretta et al. (2000), employing the hot $T_{\text {eff }}$ scale of Gratton et al. (1996) and significant NLTE corrections by Gratton et al. (1999), found that the analysis of the permitted oxygen lines results in an almost flat $[\mathrm{O} / \mathrm{Fe}] \approx 0.5$ dex (their Fig. 3), similar to that obtained from the forbidden oxygen lines.

It is not possible to perform detailed comparisons with the work of King (1993), since there is only one star (BD +02 375) in common with our work. There is a good agreement in both the $T_{\text {eff }}$ (ours is only $58 \mathrm{~K}$ higher) and $[\mathrm{O} / \mathrm{Fe}]$ of that star, but the agreement in $[\mathrm{O} / \mathrm{Fe}]$ is fortuitous, because a different $[\mathrm{Fe} / \mathrm{H}]$ and very small $\delta_{\text {NLTE }}(\mathrm{O}$ I) were employed by King (1993). Larger NLTE corrections would decrease King (1993) [O/Fe] ratios. On the other hand, $[\mathrm{O} / \mathrm{Fe}]$ should be increased due to the new low solar $A_{\mathrm{O}}$. Overall, both effects roughly cancel, preserving thus the $[\mathrm{O} / \mathrm{Fe}] \approx+0.5$ found by King (1993).

The other well-known example of a flat $[\mathrm{O} / \mathrm{Fe}]$ is that of Carretta et al. (2000), who have seven stars in common with our sample. In Figure 10 are shown the differences in $[\mathrm{Fe} / \mathrm{H}],[\mathrm{O} / \mathrm{Fe}]_{\mathrm{NLTE}}$, $A_{\mathrm{O}}^{\mathrm{LTE}}, \delta_{\mathrm{NLTE}}(\mathrm{O} \mathrm{I})$ and $T_{\text {eff. }}$. As can be seen there is a good agreement in $[\mathrm{Fe} / \mathrm{H}]$ (Figs. $10 a$ and $10 e$ ) and $T_{\text {eff }}$ (Figs. $10 d$ and $10 h)$. Our $[\mathrm{O} / \mathrm{Fe}]$ ratios are about 0.12 dex higher than theirs (Figs. $10 b$ and $10 f$ ). This is explained by the much higher solar $\mathrm{O}$ abundance $\left(A_{\mathrm{O}}=8.93\right)$ and the smaller $\delta_{\mathrm{NLTE}}(\mathrm{O} \mathrm{I})$ adopted by Carretta et al. (2000). After correcting this $(0.28 \mathrm{dex}$ in the solar $A_{\mathrm{O}}$ and 0.12 dex in $\delta_{\mathrm{NLTE}}$ ), Carretta et al. (2000) [O/Fe] ratios are only 0.04 dex smaller than ours. In fact, an excellent agreement is found in $A_{O}^{\mathrm{LTE}}$ (Figs. $10 \mathrm{c}$ and $10 \mathrm{~g}$, open circles), since it is independent of the solar $A_{\mathrm{O}}$ and $\delta_{\mathrm{NLTE}}$.

In summary, despite zero-point differences due to the adopted solar abundances and $\delta_{\mathrm{NLTE}}$, the use of a hot $T_{\text {eff }}$ scale and adequate NLTE calculations (which reproduce the new solar $\mathrm{O}$ abundance) result in a flat and low $[\mathrm{O} / \mathrm{Fe}]$ ratio in halo stars.

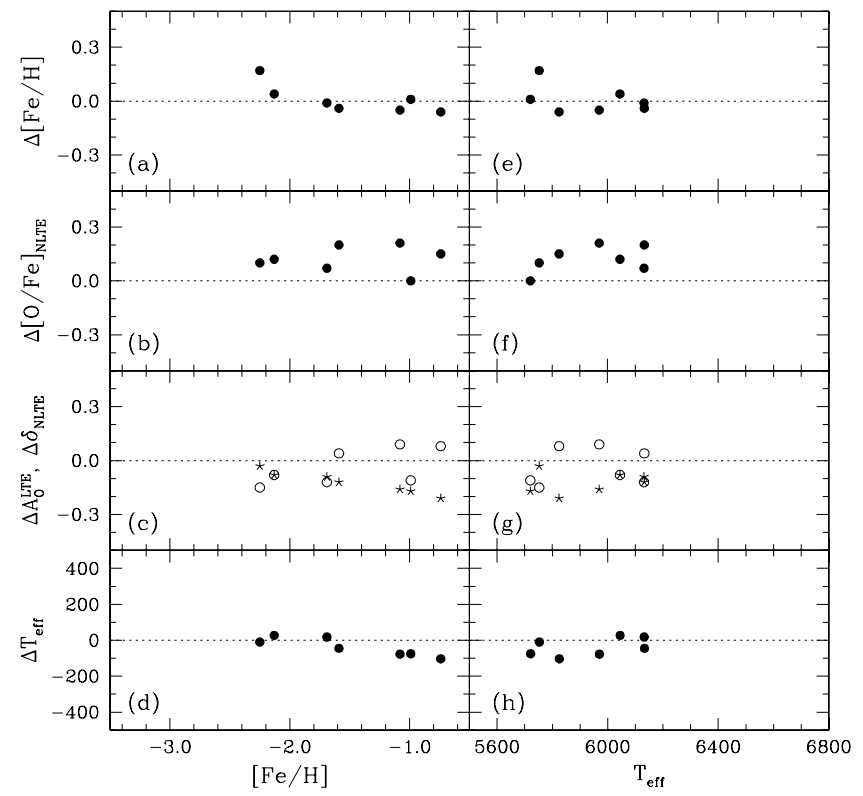

FIG. 10.-Differences between the present work and Carretta et al. (2000) are shown in the left and right panels as a function of $[\mathrm{Fe} / \mathrm{H}]$ and $T_{\text {eff }}$, respectively: $\Delta[\mathrm{Fe} / \mathrm{H}]$ ( filled circles); $\Delta[\mathrm{O} / \mathrm{Fe}]_{\mathrm{NLTE}}$ ( filled circles); $\Delta A_{O}^{\mathrm{LTE}}$ (open circles); $\Delta \delta_{\text {NLTE }}($ stars $) ; \Delta T_{\text {eff }}($ filled circles $)$.

Nevertheless, previous claims for a hotter temperature scale have not been well received by the astronomical community. The last hot $T_{\text {eff }}$ scale recently introduced in the literature is due to Fulbright $\&$ Johnson (2003), who derived a new ad hoc $T_{\text {eff }}$ scale for subgiants and giants based on forcing agreement between the oxygen abundances obtained from permitted and forbidden lines. As noted by Fulbright \& Johnson (2003), this hotter $T_{\text {eff }}$ scale for giants does not agree with theoretical isochrones, ${ }^{11}$ $T_{\text {eff }}$ from Balmer lines, and the IRFM $T_{\text {eff }}$ scale of Alonso et al. (1996, 1999). Interestingly, the ad hoc $T_{\text {eff }}$ scale leads to a mild increase of $[\mathrm{O} / \mathrm{Fe}]$ for lower metallicities, with $[\mathrm{O} / \mathrm{Fe}] \approx 0.65$ at $[\mathrm{Fe} / \mathrm{H}]=-1.5$ to $[\mathrm{O} / \mathrm{Fe}] \approx 0.8$ at $[\mathrm{Fe} / \mathrm{H}]=-2.5$.

The new $T_{\text {eff }}$ scale by RM05a and RM05b is not based on ad hoc assumptions on stellar chemical abundances. It is based on the IRFM, which is probably the least model-dependent indirect method for determining $T_{\text {eff. }}$. The problem with previous hot $T_{\text {eff }}$ scales is that they basically adopted a zero-point shift, that is, the increase in effective temperatures was applied to all stars independent of spectral type, which is not correct. Our recent studies (RM05a, RM05b) have shown that for most of the range spanned by stellar atmosphere parameters of FGK stars the IRFM $T_{\text {eff }}$ scale of Alonso et al. $(1996,1999)$ remains roughly valid, except for some regions where insufficient data was previously available, which is the case of metal-poor F and early G dwarfs.

Besides its impact on the oxygen abundances derived from the $\mathrm{O}$ I triplet, the new IRFM $T_{\text {eff }}$ scale (RM05a, RM05b) may also affect the abundances derived from other high-excitation lines (e.g., $\mathrm{C}$ I, $\mathrm{S} \mathrm{I}$ ), as well as low-excitation lines (e.g., Li I). Indeed, Meléndez \& Ramírez (2004) have shown that the use of the new IRFM $T_{\text {eff }}$ scale may be one of the factors concurring to alleviate the discrepancy between the "low" Li abundance obtained in metal-poor FG dwarfs and the "high" primordial Li abundance

\footnotetext{
${ }^{11}$ Note that we argue later that the hotter IRFM $T_{\text {eff }}$ scale of RM05a and RM05b does agree with stellar evolution models, but the $T_{\text {eff }}$ scale of RM05a and RM05b is only hotter for metal-poor turnoff stars, while the ad hoc $T_{\text {eff }}$ scale by Fulbright \& Johnson (2003) is hotter for giant stars.
} 


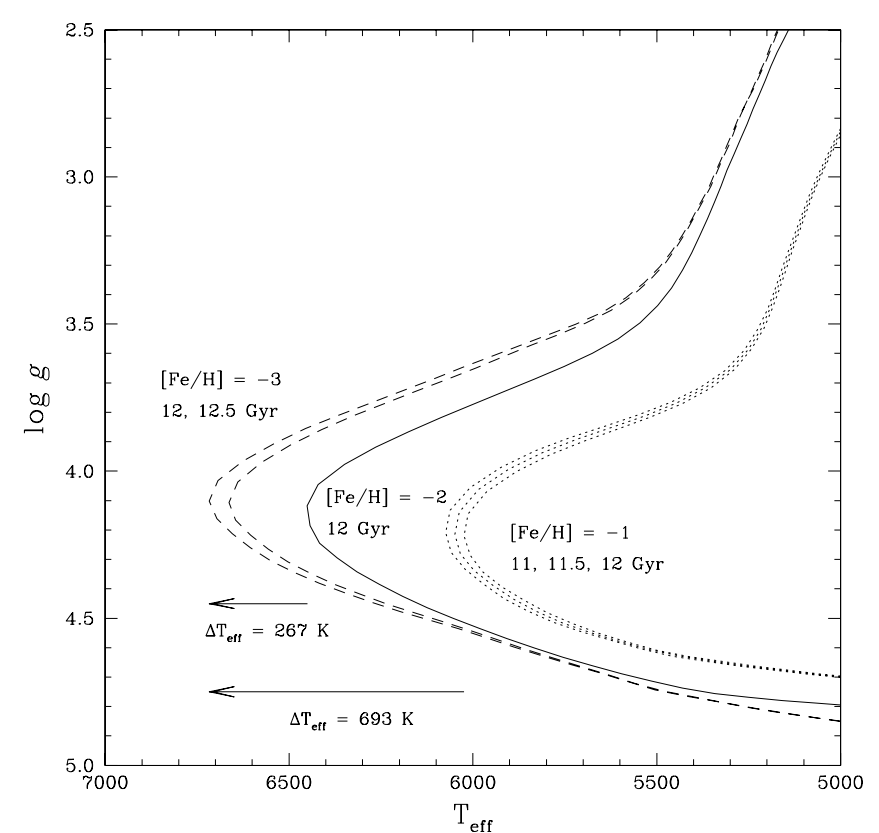

FIG. 11. $-Y^{2}$ isochrones for $[\mathrm{Fe} / \mathrm{H}]=-1$ (dotted lines), -2 (solid line), and -3 (dashed lines). The arrows show the maximum increase in $T_{\mathrm{eff}}$ of the turnoff from $[\mathrm{Fe} / \mathrm{H}]=-1$ to $-3(693 \mathrm{~K})$, and from $[\mathrm{Fe} / \mathrm{H}]=-2$ to $-3(267 \mathrm{~K})$. [See the electronic edition of the Journal for a color version of this figure.]

obtained from Wilkinson Microwave Anisotropy Probe data and big bang primordial nucleosynthesis.

\subsection{New IRFM $T_{\text {eff }}$ Scale for Halo Turnoff Stars versus Stellar Evolution}

The high effective temperatures that we obtained for metal-poor turnoff stars are supported by stellar evolution calculations, which predict that the $T_{\mathrm{eff}}$ of the turnoff strongly depends on metallicity. In Figure 11, we plot $Y^{2}$ isochrones for metallicities $[\mathrm{Fe} / \mathrm{H}]=$ $-1,-2,-3$, showing that the turnoff temperature increases up to $\approx 700 \mathrm{~K}$ from $[\mathrm{Fe} / \mathrm{H}]=-1$ to $[\mathrm{Fe} / \mathrm{H}]=-3$, assuming a constant age of 12 Gyr. Even allowing for reasonable changes in age, the $T_{\text {eff }}$ of the turnoff still strongly depends on metallicity. This metallicity dependence of the turnoff temperatures are also predicted by other stellar evolution models. The widely used Padova (e.g., Girardi et al. 2002) and Victoria (Bergbusch \& VandenBerg 2001) isochrones predict a strong dependence on metallicity, with even higher effective temperatures at the turnoff than the $Y^{2}$ isochrones. ${ }^{12}$

In order to study more quantitatively whether (or not) our IRFM $T_{\text {eff }}$ scale for turnoff stars makes sense physically, we have made a comparison of our temperatures with those predicted by stellar evolution. Since for a given metallicity the turnoff stars are the ones with highest $T_{\text {eff }}$, we divided the present sample and the sample of Meléndez \& Ramírez (2004) (which are composed mainly of main-sequence, turnoff, and subgiant stars) in metallicity bins of $0.5 \mathrm{dex}$, and adopted as turnoff IRFM $T_{\text {eff }}$ the average effective temperature of the three stars with the highest $T_{\text {eff }}$ in each metallicity bin. The results are shown in Figure 12, where we can see the impressive qualitative and quantitative agreement of the evolutionary models of metal-poor stars with the $T_{\text {eff }}$ scale by RM05a and RM05b.

\footnotetext{
12 The absolute location of the turnoff depends on the assumptions made on the stellar evolution models, especially on the mixing length. However, our main point is that the turnoff strongly depends on metallicity, and this wellestablished behavior of stellar evolution is independent of the absolute location of the turn off.
}

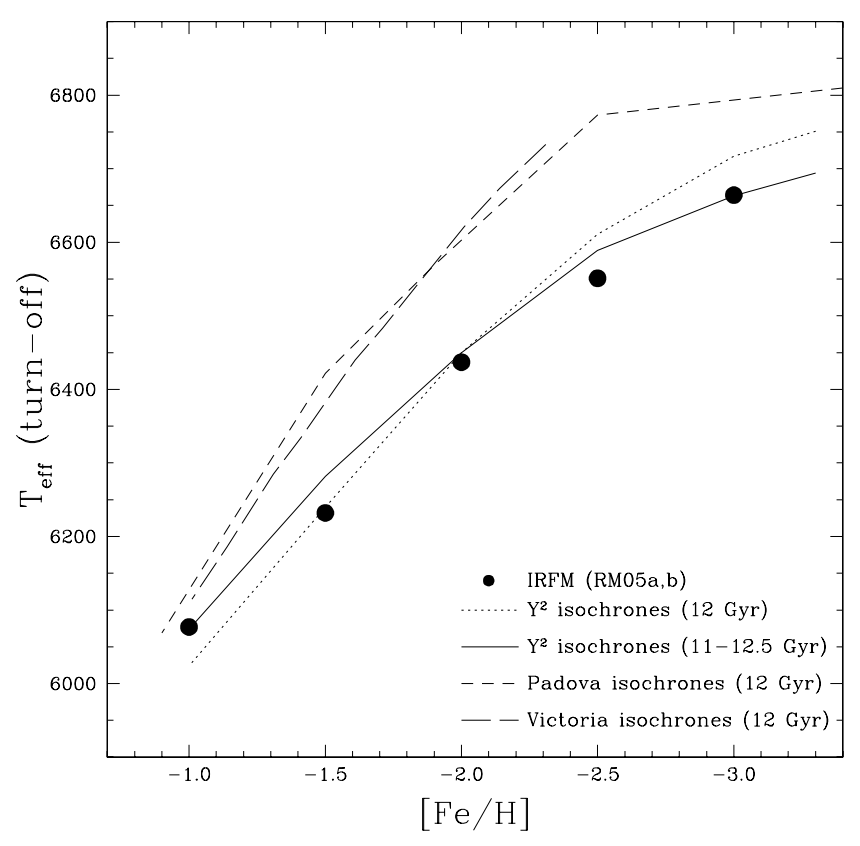

FIG. 12.-Filled circles: Temperatures of turnoff stars found in this work and in Meléndez \& Ramírez (2004), employing the IRFM $T_{\text {eff }}$ scale by RM05a and RM05b. Dotted line: Predictions of $Y^{2}$ isochrones adopting an age of $12 \mathrm{Gyr}$. Solid line: Predictions of $Y^{2}$ isochrones for ages increasing from $11 \mathrm{Gyr}([\mathrm{Fe} / \mathrm{H}]=$ $-1)$ to $12.5 \mathrm{Gyr}([\mathrm{Fe} / \mathrm{H}]=-3.3)$. Short-and long-dashed lines: $12 \mathrm{Gyr}$ turnoff $T_{\text {eff }}$ from Padova and Victoria isochrones, respectively. [See the electronic edition of the Journal for a color version of this figure.]

The metallicity dependence of the turnoff was predicted by early stellar evolution models (Simoda \& Iben 1968, 1970; Iben \& Rood 1970; Demarque et al. 1971), which showed that a decrease by a factor of 10 in metallicity (from $Z=10^{-3}$ to $10^{-4}$ ) corresponds to an increase of about $0.025 \mathrm{dex}$ in $\log T_{\text {eff }}$ (Iben $\&$ Rood 1970), that at $T_{\text {eff }}=6000 \mathrm{~K}$ corresponds to an increase in $T_{\text {eff }}$ of $355 \mathrm{~K}$ for a decrease in 1 dex in metallicity. This is due to two effects (Simoda \& Iben 1968, 1970): (1) the influence of metallicity on opacity, which contributes to an increase of the $T_{\text {eff }}$ for more metal-poor models; and (2) the influence of metallicity on the $p-p$ and CNO luminosities for low-mass stars. As evolution from the main sequence proceeds and core temperature increases, the $\mathrm{CN}$-cycle grows its importance over the $p$ - $p$ chain. The $\mathrm{CN}$-cycle does not become important until a considerable fraction of the central hydrogen has been converted into helium, which for lower metallicities occurs later, allowing the star to reach a bluer (hotter) turnoff (Iben \& Rood 1970; Simoda \& Iben 1970).

Recently, Frebel et al. (2005) announced the discovery of HE 1327-2326, a hyper-metal-poor star with $[\mathrm{Fe} / \mathrm{H}]=-5.4$, the most iron-poor star yet known. The authors argue that this star is close to the turnoff, being probably either a main-sequence or a subgiant star.

Models of hyper-metal-poor stars show that the location of the turnoff looses its sensitivity to the initial metal content for $Z$ lower than $Z \approx 10^{-6}$ (Wagner 1974; Cassisi \& Castellani 1993). That means that the $T_{\text {eff }}$ of the turnoff reaches a maximum value at $[\mathrm{Fe} / \mathrm{H}] \approx-4$. Figure 12 suggests that the turnoff $T_{\text {eff }}$ of Population III stars should be not much higher than $\approx 6700-6750 \mathrm{~K}$.

Frebel et al. (2005) determined a $T_{\text {eff }}=6180 \pm 80 \mathrm{~K}$ for HE 1327-2326, which is considerably lower than the maximum temperature allowed for a hyper-metal-poor turnoff star. Employing the Johnson-Cousin and 2MASS colors given in Aoki et al. (2006), and using $E(B-V)=0.077$, which is the same $E(B-V)$ value adopted by them, we obtained $T_{\text {eff }}=6340 \mathrm{~K}$ 
using the $T_{\text {eff }}$ scale by RM05b with $[\mathrm{Fe} / \mathrm{H}]=-3.3$. A lower metallicity must not be employed due to the low number of extremely metal-poor calibration stars. Aoki et al. (2006) found that the reddening of HE 1327-2326 may be as high as $E(B-V)=$ 0.104 , as estimated from the interstellar Na I D2 line. Adopting $E(B-V)=0.104, T_{\text {eff }}=6500 \mathrm{~K}$ is obtained from the $T_{\text {eff }}$ scale of RM05b. Aoki et al. (2006) have found that the spectroscopic temperature (based on Balmer lines and the HP2 index) of HE $1327-2326$ is about $200-300 \mathrm{~K}$ lower than G64-12. Using our $T_{\text {eff }}$ for G64-12, implies that the spectroscopic $T_{\text {eff }}$ of $\mathrm{HE}$ $1327-2326$ is about $6360-6460 \mathrm{~K}$.

It is of the uttermost importance to determine a good parallax for HE 1327-2326, in order to assess the evolutionary stage of this hyper-metal-poor star, and to further constraint its $T_{\text {eff. }}$. For example, a trigonometric gravity of $\log g=4.1 \pm 0.1$ dex would constraint HE $1327-2326$ to the turnoff (within $\approx 200 \mathrm{~K}$ ), and if this star turns out to be hotter than previously thought, then the obtained abundances and their interpretation might require revision. This is especially important for the abundance of elements very sensitive to $T_{\text {eff }}$, like the oxygen abundance determined from UV OH lines.

\section{CONCLUSIONS}

We have determined oxygen and iron abundances in 31 metalpoor $(-3.2<[\mathrm{Fe} / \mathrm{H}]<-0.7)$ stars close to the turnoff, employing high-resolution high-S/N UVES data taken from Ake04 and N04.

We find a flat $[\mathrm{O} / \mathrm{Fe}]=+0.5$, independent of metallicity, temperature and surface gravity in the ranges $-3.2<[\mathrm{Fe} / \mathrm{H}]<$ $-0.7,5700 \mathrm{~K}<T_{\text {eff }}<6700 \mathrm{~K}$, and $3.7<\log g<4.5$, respectively. Our work confirms previous studies (e.g., Carretta et al. 2000; Primas et al. 2001), which have already found a flat and low $[\mathrm{O} / \mathrm{Fe}]$ ratio in halo dwarfs from the $\mathrm{O}$ I triplet, extending the constancy of the $[\mathrm{O} / \mathrm{Fe}]$ ratios down to $[\mathrm{Fe} / \mathrm{H}]=-3.2$.

The flat $[\mathrm{O} / \mathrm{Fe}]$ ratio is mainly due to the use of adequate NLTE calculations and the new IRFM $T_{\text {eff }}$ scale by RM05a and RM05b, which for metal-poor turnoff stars is hotter than most previous $T_{\text {eff }}$ scales available in the literature. We find a low starto-star scatter of $0.136 \mathrm{dex}$ for the whole sample, or $\sigma=0.10 \mathrm{dex}$ for the sample with low reddening uncertainty.

The observed star-to-star scatter $\left(\sigma_{\text {obs }}=0.136 \mathrm{dex}\right)$ is almost completely explained by errors in the analysis $(\approx 0.121 \mathrm{dex})$, leaving little room for intrinsic scatter. Hence, the Galaxy was extremely efficient in mixing the chemical elements ejected by supernovae. Other recent works in the literature have also found very small star-to-star scatter for other $\alpha$-elements (e.g., Cayrel et al. 2004; Cohen et al. 2004; Arnone et al. 2005). Furthermore, the low scatter implies a small contribution to the halo from metalpoor stars that originated in $\mathrm{dSph}$ galaxies, since much lower $[\alpha / \mathrm{Fe}]$ ratios are commonly seen in such galaxies (e.g., Venn et al. 2004; see also discussion and references given in Catelan 2006).

The constancy of the $[\mathrm{O} / \mathrm{Fe}]$ ratio over more than 2 orders of magnitude in $[\mathrm{Fe} / \mathrm{H}]$, from $[\mathrm{Fe} / \mathrm{H}]=-0.7$ to $[\mathrm{Fe} / \mathrm{H}]=-3.2$, is telling us that the formation of the halo was extremely fast, with a timescale shorter than the bulk of Type Ia SNe. Our data provides tight constraints for Galactic chemical evolution models (e.g., Matteucci \& Recchi 2001; Alibés et al. 2001; Goswami \& Prantzos 2000; Portinari et al. 1998; Samland 1998; Chiappini et al. 1997).

Our low $[\mathrm{O} / \mathrm{Fe}]=+0.5$ constrains the ${ }^{6} \mathrm{Li}$ production by GCR models, which are not able to explain the detection of ${ }^{6} \mathrm{Li}$ in a star with $[\mathrm{Fe} / \mathrm{H}]=-2.7$ (Asplund et al. 2006), hence reinforcing the case for a pre-Galactic origin of the recent ${ }^{6} \mathrm{Li}$ detections in very metal-poor stars.

Recent determinations of $\mathrm{H} \alpha$ temperatures in turnoff stars by Asplund et al. (2006) are in very good relative agreement with the RM05b $T_{\text {eff }}$ scale in the metallicity range $-2.7<[\mathrm{Fe} / \mathrm{H}]<-1$, with a zero-point difference of only $61 \pm 62 \mathrm{~K}$.

As shown in Figures 11 and 12, the strong metallicity dependence of the temperature of turnoff stars is not unphysical, but a natural consequence of stellar evolution. I. Ramírez et al. (2006, in preparation), has recently confirmed the hot $T_{\text {eff }}$ scale of RM05b for one star, BD +174708 , by fitting its observed absolute flux distribution from Hubble Space Telescope observations with Kurucz models. Indeed, the $T_{\text {eff }}$ obtained by Ramírez is about $100 \mathrm{~K}$ higher than the $T_{\text {eff }}$ obtained in this work with the $T_{\text {eff }}$ scale of RM05b. This is not in conflict with stellar evolution calculations, since there is still room for an increase of about $100 \mathrm{~K}$ in the RM05b $T_{\text {eff }}$ scale of metal-poor turnoff stars (Fig. 12).

In the future, it would be important to take into account also granulation effects, performing full 3D+NLTE analyses. This is the way to go for future abundance studies. Also, it is very important to perform future verifications of the $T_{\text {eff }}$ scale of RM $05 \mathrm{~b}$.

J. M. thanks A. McWilliam and C. Allende Prieto for providing Fortran and IDL codes to interpolate model atmospheres, thanks partial support from NSF grant AST 02-05951 to J. G. Cohen, and acknowledges the support of the American Astronomical Society and the NSF in the form of two International Travel Grants. I. R. acknowledges support from the Robert A. Welch Foundation of Houston, Texas to D. Lambert. We thank an anonymous referee for useful suggestions; K. A. Olive and B. D. Fields for sending in electronic form data plotted in Fields et al. (2005); M. Catelan for his comments, especially on stellar evolution; C. Chiappini for comments on early results; J. G. Cohen and C. Dickinson for comments and proofreading of an early version of the manuscript; $M$. Asplund for useful comments, especially for confirming errors in the NLTE corrections of the most metal-poor stars in Ake04; A. E. García-Pérez for sending a preprint prior to publication; P. Nissen for clarifying the solar $A_{\mathrm{Fe}}$ of Nissen et al. (2002) and N04; and D. Fabbian for useful discussions on NLTE effects. This research has been funded partially by the Spanish Ministerio de Educación y Ciencia through project AYA2004-05792 and by the European Commission through INTAS grant 00-00084. We have made use of data from the following: the Hipparcos astrometric mission of the ESA; UVES/VLT of ESO; SIMBAD database operated at CDS; and 2MASS of the University of Massachusetts and IPAC/Caltech, funded by NASA and NSF.
Abia, C., \& Rebolo, R. 1989, ApJ, 347, 186

Akerman, C. J., Carigi, L., Nissen, P. E., Pettini, M., \& Asplund, M. 2004, A\&A, 414, 931 (Ake04)

Alibés, A., Labay, J., \& Canal, R. 2001, 370, 1103

Allende Prieto, C., Asplund, M., \& Fabiani Bendicho, P. 2004, A\&A, 423, 1109
Alonso, A., Arribas, S., \& Martínez-Roger, C. 1996, A\&A, 313, 873

1999, A\&AS, 140, 261

Aoki, W., et al. 2006, ApJ, 639, 897

Arce, H. G., \& Goodman, A. A. 1999, ApJ, 512, L135

Arenou, F., Grenon, M., \& Gomez, A. 1992, A\&A, 258, 104 (A92) 
Arnone, E., Ryan, S. G., Argast, D., Norris, J. E., \& Beers, T. C. 2005, A\&A, 430, 507

Asplund, M. 2005, ARA\&A, 43, 481

Asplund, M., \& García-Pérez, A. E. 2001, A\&A, 372, 601

Asplund, M., Grevesse, N., Sauval, A. J., Allende Prieto, C., \& Kiselman, D. 2004, A\&A, 417, 751 (Asp04)

Asplund, M., Gustafsson, B., Kiselman, D., \& Eriksson, K. 1997, A\&A, 318, 521

Asplund, M., Lambert, D., Nissen, P. E., Primas, F., \& Smith, V. 2006, ApJ, in press (astro-ph/0510636)

Asplund, M., Nordlund, Å., Trampedach, R., \& Stein, R. F. 2000, A\&A, 359, 743

Aufdenberg, J. P., Ludwig, H.-G., \& Kervella, P. 2005, ApJ, 633, 424

Axer, M., Fuhrmann, K., \& Gehren, T. 1994, A\&A, 291, 895

Barbuy, B. 1988, A\&A, 191, 121

Barklem, P. S., \& Aspelund-Johansson, J. 2005, A\&A, 435, 373

Barklem, P. S., Piskunov, N., \& O’Mara, B. J. 2000, A\&AS, 142, 467

Bergbusch, P. A., \& VandenBerg, D. A. 2001, ApJ, 556, 322

Bessell, M. S., Sutherland, R. S., \& Ruan, K. 1991, ApJ, 383, L71

Boesgaard, A. M., King, J. R., Deliyannis, C. P., \& Vogt, S. S. 1999, AJ, 117, 492

Bond, H. E. 1980, ApJS, 44, 517

Breitschwerdt, D., Freyberg, M. J., \& Egger, R. 2000, A\&A, 364, 935

Burstein, D. 2003, AJ, 126, 1849

Burstein, D., \& Heiles, C. 1978, ApJ, 225, 40

Carlsson, M., \& Judge, P. G. 1993, ApJ, 402, 344

Carney, B. W. 1983, AJ, 88, 623

Carretta, E., Gratton, R. G., \& Sneden, C. 2000, A\&A, 356, 238

Cassisi, S., \& Castellani, V. 1993, ApJS, 88, 509

Castelli, F., Gratton, R. G., \& Kurucz, R. L. 1997, A\&A, 324, 432

Castelli, F., \& Kurucz, R. L. 2003, in IAU Symp. 210, Modelling of Stellar Atmospheres, ed. N. Piskunov, W. W. Weiss \& D. F. Gray (San Frascisco: ASP), 20

Catelan, M. 2006, in ASP Conf. Ser., Resolved Stellar Populations, ed. D. VallsGabaud \& M. Chavez (San Francisco: ASP), in press (astro-ph/0507464)

Cavallo, R. M., Pilachowski, C. A., \& Rebolo, R. 1997, PASP, 109, 226

Cayrel, R., et al. 2004, A\&A, 416, 1117

Chen, B., Vergely, J. L., Valette, B., \& Carraro, G. 1998, A\&A, 336, 137 (C98)

Chen, B., et al. 1999, A\&A, 352, 459

Chiappini, C., Matteucci, F., \& Gratton, R. 1997, ApJ, 477, 765

Chiappini, C., Romano, D., \& Matteucci, F. 2003 MNRAS, 339, 63

Cohen, J., et al. 2004, ApJ, 612, 1107

Crawford, D. L. 1975, PASP, 87, 481

Demarque, P., Mengel, J. G., \& Aizenman, M. L. 1971, ApJ, 163, 37

Demarque, P., Woo, J.-H., Kim, Y.-C., \& Yi, S. K. 2004, ApJS, 155, 667

Dutra, C. M., Ahumada, A. V., Clariá, J. J., Bica, E., \& Barbuy, B. 2003a, A\&A, 408, 287

Dutra, C. M., Santiago, B. X., Bica, E., \& Barbuy, B. 2003b, MNRAS, 338, 253

ESA. 1997, The Hipparcos and Tycho Catalogues (ESA SP-1200; Noordwijk: ESA

Frebel, A., et al. 2005, Nature, 434, 871

Fields, B. D., \& Olive, K. A. 1999, ApJ, 516, 797

Fields, B. D., Olive, K. A., \& Vangioni-Flam, E. 2005, ApJ, 623, 1083

Fitzgerald, M. P. 1968, AJ, 73, 983

Fulbright, J. P., \& Johnson, J. A. 2003, ApJ, 595, 1154

Fulbright, J. P., \& Kraft, R. P. 1999, AJ, 118, 527

García Pérez, A. E., Asplund, M., Primas, F., Nissen, P. E., \& Gustafsson, B. 2006, A\&A, in press (astro-ph/0512290)

Gehren, T., Korn, A. J., \& Shi, J. 2001, A\&A, 380, 645

Girardi, L., et al. 2002, A\&A, 391, 195

Goswami, A., \& Prantzos, N. 2000, A\&A, 359, 191

Gratton, R. G., Carretta, E., \& Castelli, F. 1996, A\&A, 314, 191

Gratton, R. G., Carretta, E., Eriksson, K., \& Gustafsson, B. 1999, A\&A, 350, 955

Gurtovenko, E. A., \& Kostik, R. I. 1989, Fraunhofer Spectrum and the System of Solar Oscillator Strenghts (Kiev: Noukova Dumka) (GK89)

Hakkila, J., Myers, J. M., Stidham, B., \& Hartmann, D. H. 1997, AJ, 114, 2043 (H97)

Hannaford, P., Lowe, R. M., Grevesse, N., \& Noels, A. 1992, A\&A, 259, 301 (H92)

Hinkle, K., Wallace, L., Valenti, J., \& Harmer, D. 2000, Visible and Near Infrared Atlas of the Arcturus Spectrum, 3727-9300 ̊̊ (San Francisco: ASP Holweger, H., \& Müller, E. A. 1974, Sol. Phys. 39, 19 (HM74)

Hudson, M. J. 1999, PASP, 111, 57

Iben, I., Jr., \& Rood, R. T. 1970, ApJ, 159, 605

Israelian, G., García López, R. J., \& Rebolo, R. 1998, ApJ, 507, 805
Israelian, G., Rebolo, R., García López, R. J., Bonifacio, P., Molaro, P., Basri, G., \& Shchukina, N. 2001, ApJ, 551, 833 (erratum 560, 535)

Israelian, G., Shchukina, N., Rebolo, R., Basri, G., González Hernández, J. I., \& Kajino, T. 2004, A\&A, 419, 1095

Jedamzik, K. 2000, Phys. Rev. Lett., 84, 3248 2004, Phys. Rev. D, 70, 063524

Kim, Y.-C., Demarque, P., Yi, S. K., \& Alexander, D. R. 2002, ApJS, 143, 499

King, J. R. 1993, AJ, 106, 1206 2000, AJ, 120, 1056

Kostik, R. I., Shchukina, N. G., \& Rutten, R. J. 1996, A\&A, 305, 325

Kurucz, R. L., Furenlid, I., Brault, J., \& Testerman, L. 1984, Solar Flux Atlas from 296 to $1300 \mathrm{~nm}$ (Sunspot: NSO) (K84)

Lallemnet, R., Welsh, B. Y., Vergely, J. L., Crifo, F., \& Sfeir, D. 2003, A\&A, 411,447

Lambert, D. L. Heath, J. E., Lemke, M., \& Drake, J. 1996, ApJS, 103, 183

Leroy, J. L. 1999, A\&A, 346, 955

Masseron, T., Plez, B., Primas, F., et al. 2005, in IAU Symp. 228, From Lithium to Uranium: Elemental Tracers of Early Cosmic Evolution, ed. V. Hill, P. François \& F. Primas (Cambridge: Cambridge Univ. Press), 219

Matteucci, F., \& Recchi, S. 2001, ApJ, 558, 351

McWilliam, A. 1997, ARA\&A, 35, 503

Meléndez, J. 2004, ApJ, 615, 1042

Meléndez, J., \& Barbuy, B. 2002, ApJ, 575, 474

Meléndez, J., Barbuy, B., \& Spite, F. 2001, ApJ, 556, 858

Meléndez, J., \& Ramírez, I. 2004, ApJ, 615, L33

Mermilliod, J.-C., Mermilliod, M., \& Hauck, B. 1997, A\&AS, 124, 349

Mishenina, T. V., Korotin, S. A., Klochkova, V. G., \& Panchuk, V. E. 2000, A\&A, 353, 978

Neckel, Th., \& Klare, G. 1980, A\&AS, 42, 251

Nissen, P. E. 1994, Rev. Mex. AA, 29, 129

Nissen, P. E., Chen, Y. Q., Asplund, M., \& Pettini, M. 2004, A\&A, 415, 993 (N04)

Nissen, P. E., Primas, F., Asplund, M., \& Lambert, D. L. 2002, A\&A, 390, 235

Portinari, L., Chiosi, C., \& Bressan, A. 1998, A\&A, 334, 505

Prantzos, N. 2006, A\&A, 448, 665

Prantzos, N., Cassé, M., \& Vangioni-Flam, E. 1993, ApJ, 403, 630

Primas, F., et al. 2001, NewA Rev., 45, 541

Ramaty, R., Scully, S. T., Lingenfelter, R. E., \& Kozlovsky, B. 2000, ApJ, 534, 747

Ramírez, I., \& Meléndez, J. 2005a, ApJ, 626, 446 (RM05a) $2005 \mathrm{~b}$, ApJ, 626, 465 (RM05b)

Rollinde, E., Vangioni-Flam, E., \& Olive, K. A. 2005, ApJ, 627, 666

Samland, M. 1998, ApJ, 496, 155

Scalo, J., \& Elmegreen, B. G. 2004, ARA\&A, 42, 275

Schlegel, D. J., Finkbeiner, D. P., \& Davis, M. 1998, ApJ, 500, 525 (S98)

Schnabel, R., Kock, M., \& Holweger, H. 1999, A\&A, 342, 610

Schnabel, R., Schultz-Johanning, M., \& Kock, M. 2004, A\&A, 414, 1169

Schuster, W. J., \& Nissen, P. E. 1989, A\&A, 221, 65

Sfeir, D. M., Lallemnet, R., Crifo, F., \& Welsh, B. Y. 1999, A\&A, 346, 785

Shchukina, N., \& Trujillo Bueno, J. 2001, ApJ, 550, 970

Shchukina, N., Trujillo Bueno, J., \& Asplund, M. 2005, ApJ, 618, 939

Shchukina, N., Vasiljeva, I. E., Trujillo Bueno, J., \& Asplund, M. 2003, in IAU Symp. 210, Modelling of Stellar Atmospheres, ed. N. Piskunov, W. W. Weiss, \& D. F. Gray (San Francisco: ASP), 10

Simoda, M., \& Iben, I., Jr. 1968, ApJ, 152, 509

. 1970, ApJS, 22, 81

Sneden, C. 1973, Ph.D. thesis, Univ. Texas

Sneden, C., Kraft, R. P., Langer, G. E., Prosser, C. F., \& Shetrone, M. D. 1994, AJ, 107, 1773

Sneden, C., Kraft, R. P., Prosser, C. F., \& Langer, G. E. 1991, AJ, 102, 2001

Sneden, C., \& Primas, F. 2001, NewA Rev., 45, 513

Spite, M., \& Spite, F. 1991, A\&A, 252, 689

Takeda, Y. 2003, A\&A, 402, 343

Thévenin, F., \& Idiart, T. 1999, ApJ, 521, 753

Tinsley, B. 1979, ApJ, 229, 1046

Tomkin, J., Lemke, M., Lambert, D. L., \& Sneden, C. 1992, AJ, 104, 1568

Vangioni-Flam, E., Cassé, M., \& Audouze, J. 2000, Phys. Rep., 333, 365

Venn, K. A., Irwin, M., Shetrone, M. D., Tout, C. A., Hill, V., \& Tolstoy, E. 2004, AJ, 128, 1177

Vergely, J.-L., Ferrero, R. F., Egret, D., \& Koeppen, J. 1998, A\&A, 340, 543

Wagner, R. L. 1974, ApJ, 191, 173

Wheeler, J. C., Sneden, C., \& Truran, J. W., Jr. 1989, ARA\&A, 27, 279

Wiese, W. L., Fuhr, J. R., \& Deters, T. M. 1996, Atomic Transition Probabilities of Carbon, Nitrogen, and Oxygen (Washington: American Chem. Soc.) 